\title{
Smart composites with tunable stress-strain curves
}

\author{
M. Özcan ${ }^{1} \cdot$ M. Cakmakci ${ }^{1} \cdot$ i. Temizer ${ }^{1}$ \\ Received: 12 February 2019 / Accepted: 20 September 2019 / Published online: 8 October 2019 \\ (c) Springer-Verlag GmbH Germany, part of Springer Nature 2019
}

\begin{abstract}
Smart composites with tunable stress-strain curves are explored in a numerical setting. The macroscopic response of the composite is endowed with tunable characteristics through microscopic constituents which respond to external stimuli by varying their elastic response in a continuous and controllable manner. This dynamic constitutive behavior enables the composite to display characteristics that cannot be attained by any combination of traditional materials. Microscopic adaptation is driven through a repetitive controller which naturally suits the class of applications sought for such composites where loading is cyclic. Performance demonstrations are presented for the overall numerical framework over complex paths in macroscopic stress-strain space. Finally, representative two- and three-dimensional tunable microstructures are addressed by integrating the control approach within a computational environment that is based on the finite element method, thereby demonstrating the viability of designing and analyzing smart composites for realistic applications.
\end{abstract}

Keywords Micromechanics $\cdot$ Composites $\cdot$ Smart materials $\cdot$ Adaptivity $\cdot$ Control theory

\section{Introduction}

Composite materials have led to unprecedented design and performance capabilities in many areas of engineering, a prime example being carbon fiber composites that were introduced in the 1960s [1]. Today, they enable product design in aerospace, automotive, sports and medical industries which are energy efficient, strong and lightweight. Despite the proven success of a variety of composite materials, they typically cannot adapt to varying performance criteria because their microstructures are static with respect to both morphological as well as mechanical properties. In other words, their microscopic properties cannot evolve so as to meet demands which differ from the initial design phase or to deliver non-trivial macroscopic thermomechanical responses that are too complex for any combination of traditional materials. The exploration of a class of composites with dynamic microstructures which can achieve such variable target behavior constitutes the focus of this work.

The main premise of composite materials is that their macroscopic response may be tailored by altering the microstructure so as to meet desired performance criteria,

İ. Temizer

temizer@bilkent.edu.tr

1 Department of Mechanical Engineering, Bilkent University, 06800 Ankara, Turkey ideally attempting to achieve an optimal microstructure that delivers the best response among all alternatives under these criteria while satisfying design constraints such as volume fraction. One major class of composites involves particles or fibers embedded in a matrix material, and the design procedure attempts to determine the optimal particle morphology [2,3] or fiber orientation [4,5]. Another major class of composites, including porous ones, rely on more recent manufacturing techniques which enable large scale production of materials with intricate periodic microstructures [6]. The computational design of such microstructures is often realized through topology optimization techniques $[7,8]$ and can deliver non-traditional macroscopic responses such as a negative thermal expansion coefficient or Poisson's ratio [9-11] in addition to the possibility of meeting macroscopic performance criteria such as maximal stiffness at the point of application of a force when these tailored materials are employed in structural applications $[7,12,13]$.

When the macroscale performance criteria are fixed, the design methodology for the types of composites mentioned above is expected to deliver the optimal microstructure that ensures the best response possible within the search space. However, if a design criterion varies with time, for instance when the direction of the force applied on the structure changes continuously, the initially optimal microstructure may be significantly sub-optimal by the time the structural 


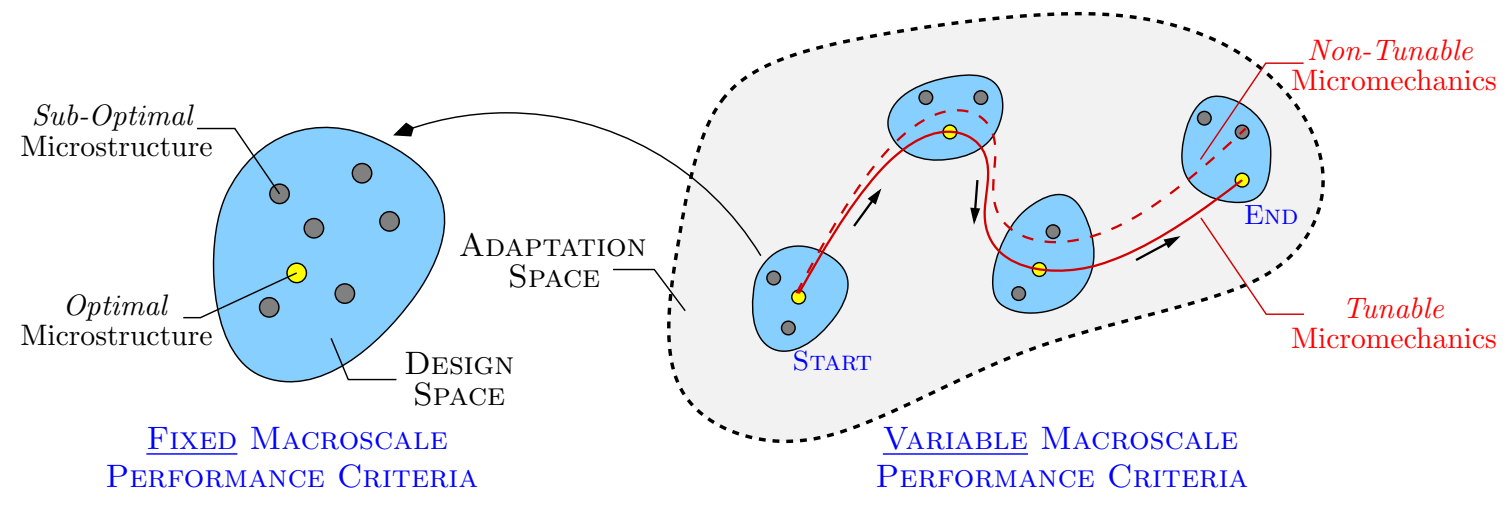

Fig. 1 Microstructure design algorithms typically operate under an objective function that reflects fixed macroscale performance criteria. However, the optimal design will perform increasingly sub-optimally if used under an objective function which starts with the original one and evolves towards an entirely different one. If the microstructure is additionally tunable, it can adapt to the varying performance demands in order to ensure (nearly) optimal response at all times process ends. This points out a shortcoming of such static microstructures and highlights the need for dynamic ones which can adapt to the changing criteria so as to ensure that the microstructure remains (nearly) optimal at all times, as depicted in Fig. 1, thereby effectively rendering the composite smart. This might be possible, for instance, if (1) the microstructure topology or (2) the microscopic constitutive behavior can evolve in a controllable and continuous manner through external stimuli, in other words if the microstructure is tunable. It is important to point out that the evolution should be controllable for the purposes of this study, in other words there should be a microstructural process that can be activated independently from the macroscopic process which leads to changing performance criteria. For instance, a microstructure can change its topology progressively with increasing load without any external stimulus $[14,15]$ but this change has no control degree of freedom that is independent from the loading process. Also, in order to adapt to continuously varying criteria, the microstructure must also be able to respond continuously to the stimulus. For instance, if it adapts its topology to a certain degree only in a preprocessing stage but remains fixed afterwards [16] then it cannot ensure an optimal response at all times. Both of these examples were in the context of topology adaptation. The focus of this work, however, will be on tunable constitutive behavior as will be discussed further below in a purely mechanical setting so that the aim will be to control the path in the macroscopic stressstrain space. Clearly, a higher degree of freedom in tuning may be achieved by combining topological and constitutive adaptation which, however, is beyond the scope of this work. Additionally, as will be pointed out through various examples, microstructure topology design can also be beneficial in constitutive adaptation in microstructures, although this will not be explored presently. Finally, the degree of freedom offered by adaptation should ideally be large enough to encompass the optimal response at all times. Cases when this condition is not met will be discussed.

An important ingredient of the idea discussed above is a microstructural constituent with tunable mechanical constitutive response, presently confined to solid materials. There are a variety of novel materials which respond to external stimuli such as heat in a reversible but on-off manner $[17,18]$. However, continuity in tunability is lacking in such responses. Magnetorheological elastomers stand out as a particularly suitable candidate for the purposes of this study due the clearly observable continuous influence of the magnetic field on the stress-strain curve under dynamic loading $[19,20]$. Consequently, they are suitable for application in tunable mechanical and structural components such as actuators under cyclic loading [21-23]. Indeed, tunable materials and smart composites can provide alternative means of achieving actuation in robotics and aerospace where there is a need for tunable stiffness $[24,25]$ with the magnetic field as a particularly suitable method for inducing actutation in the presence of intricate geometries [26,27], which further highlights the underlying motivation of this work from a broad perspective.

The precise goal of this work can now be stated as the development of the numerical framework that is necessary to work with tunable composites in practice and the simultaneous demonstration of the capabilities which are offered by such materials. This work constitutes the first study in the literature with respect to both of these aspects to the best of authors' knowledge. For this purpose, the micromechanical background, the major ingredients for tunable mechanics and indicative examples for how control is achieved are outlined in Sect. 2 in a one-dimensional setting. In particular, although linear elasticity is assumed throughout most of this work, an example based on viscoelasticity demonstrates the applicability of the framework to inelastic behavior that underlies 
tunable materials such as magnetorheological elastomers. Subsequently, the choice of the controller which is central to the numerical framework is discussed compactly in Sect. 3 with a focus on the non-standard aspects of control theory that have been adapted towards the purposes of this study. Finally, the integration of the controller within a finite element method (FEM) environment is carried out in Sect. 4 where various examples will demonstrate the feasibility of attaining tunable mechanics when the microstructure is complex enough to require the computational determination of the microscopic stress field. The study is then concluded with a summary of the challenges and recommendations for future work.

\section{Mechanics of smart composites}

\subsection{Macroscopic response}

\subsubsection{Average stress-strain relation}

The macroscopic response of heterogeneous materials with a periodic or random microstructure can be expressed approximately through micromechanical models or more accurately through homogenization theory [28-30]. In this work, the focus will be on periodic microstructures that are particularly suitable for a homogenization-based analysis. The domain of the unit-cell of periodicity (see Fig. 2) will be denoted by $\mathcal{Y}$ and, for a generic variable $\mathcal{Q}$, cell-averaging over $\mathcal{Y}$ by $\langle\mathcal{Q}\rangle=|\mathcal{Y}|^{-1} \int_{\mathcal{Y}} \mathcal{Q} \mathrm{d} v$. It will be assumed that the microstructure is composed of $M$ distinct constituents, each occupying a domain $\mathcal{Y}^{(I)} \subset \mathcal{Y}$, with a corresponding averaging operator $\langle\mathcal{Q}\rangle^{(I)}=\left|\mathcal{Y}^{(I)}\right|^{-1} \int_{\mathcal{Y}^{(I)}} \mathcal{Q} \mathrm{d} v$ and a cell fraction $f^{(I)}=\left|\mathcal{Y}^{(I)}\right| /|\mathcal{Y}|$. One therefore has the relation

$$
\langle\mathcal{Q}\rangle=\sum_{I=1}^{M} f^{(I)}\langle\mathcal{Q}\rangle^{(I)} .
$$

If the quantity $\mathcal{Q}$ happens to be a constant over $\mathcal{Y}^{(I)}$, it will be indicated with $\mathcal{Q}^{(I)}$ so that $\langle\mathcal{Q}\rangle=\sum_{I=1}^{M} f^{(I)} \mathcal{Q}^{(I)}$. The particular distribution of the constituents over $\mathcal{Y}$ will be referred to as the microstructure topology.

Of particular interest are the microscopic stress $(\sigma)$ and strain $(\boldsymbol{\epsilon})$ distributions that are induced when the unit-cell is subjected to boundary conditions which are relevant to homogenization. Their macroscopic counterparts $(\overline{\boldsymbol{\sigma}}$ and $\overline{\boldsymbol{\epsilon}})$ are defined through cell-averaging:

$\bar{\sigma}=\langle\sigma\rangle, \quad \bar{\epsilon}=\langle\epsilon\rangle$.

Throughout most of this work, attention will be focused to an elastic response at the small deformation regime- exceptions will be discussed. When the microscopic response is linearly elastic, $\boldsymbol{\sigma}(t)=\boldsymbol{I E} \boldsymbol{\epsilon}(t)$ holds where the microscopic elasticity tensor $\boldsymbol{I E}$ is a constant $\boldsymbol{I E}^{(I)}$ over each $\mathcal{Y}^{(I)}$ and $t$ denotes a possible dependence on time due to temporal variations in the boundary conditions on the unit-cell. In this case, the macroscopic response may be explicitly stated as $\overline{\boldsymbol{\sigma}}(t)=\overline{\boldsymbol{E}} \overline{\boldsymbol{\epsilon}}(t)$ where $\overline{\boldsymbol{I E}}$ is the macroscopic elasticity tensor. It is important to highlight that this macroscopic relation holds as long as the microscopic response is linearly elastic, even if $\boldsymbol{I E}^{(I)}$ are also not fixed but rather vary over time. In other words, temporal variations in $\boldsymbol{I E}^{(I)}$ cause temporal variations in $\overline{\boldsymbol{I E}}$ but $\overline{\boldsymbol{\sigma}}(t)=\overline{\boldsymbol{I E}}(t) \overline{\boldsymbol{\epsilon}}(t)$ always holds where the determination of $\overline{\boldsymbol{I E}}$ at any given time instant $t$ is subject to the classical methods of homogenization based on the $\boldsymbol{I E}^{(I)}(t)$ values. Also note that within these methods, the determination of $\overline{\boldsymbol{I E}}$ requires the solution of multiple cell problems [28]. As a consequence, the inverse problem of determining the optimal values of $\boldsymbol{I E}^{(I)}$ in order to obtain a desired $\overline{\boldsymbol{I E}}$ is not straightforward, even for a fixed microstructure topology.

\subsubsection{One-dimensional setting}

The control framework will initially be developed in a single-input-single-output (SISO) setting. Although a uniaxial loading setup can be constructed for this purpose, a one-dimensional setting will be considered that is motivated by classical layered composites with isotropic constituents (see also Sect. 3.2). In such a scenario, depending on whether the loading axis is parallel $(\|)$ or perpendicular $(\perp)$ to the layers, the macroscopic elastic modulus $\bar{E}$ which satisfies $\bar{\sigma}=\bar{E} \bar{\epsilon}$ may be expressed in terms of the elastic moduli $E^{(I)}$ of the constituents:

$$
\bar{E}_{\|}=f^{(1)} E^{(1)}+f^{(2)} E^{(2)}, \quad \bar{E}_{\perp}=\left(f^{(1)} / E^{(1)}+f^{(2)} / E^{(2)}\right)^{-1} .
$$

Eventually, from a control perspective, $\bar{E}_{\|}$will induce a linear framework whereas $\bar{E}_{\perp}$ will induce a nonlinear framework that will help demonstrate particular challenges. Note that in this case, the stress and strain will both be constants over each constituent $\left(\sigma^{(I)}=E^{(I)} \epsilon^{(I)}\right)$ where $\epsilon^{(I)}=\bar{\epsilon}$ for parallel loading and $\sigma^{(I)}=\bar{\sigma}$ for perpendicular loading. It should be emphasized that in a multi-dimensional setting (Sect. 4) the macroscopic elasticity tensor $\overline{\boldsymbol{I E}}$ discussed earlier will never be computed because of its computational expense. Instead, the only quantity of interest will be the macroscopic stress $\bar{\sigma}$, which is obtained through cell-averaging after solving for the microscopic stress field. In the one-dimensional setting based on the chosen classical composite models, the determination of $\sigma$ for cell-averaging towards $\bar{\sigma}$ is effectively equivalent to directly calculating $\bar{E}$. 


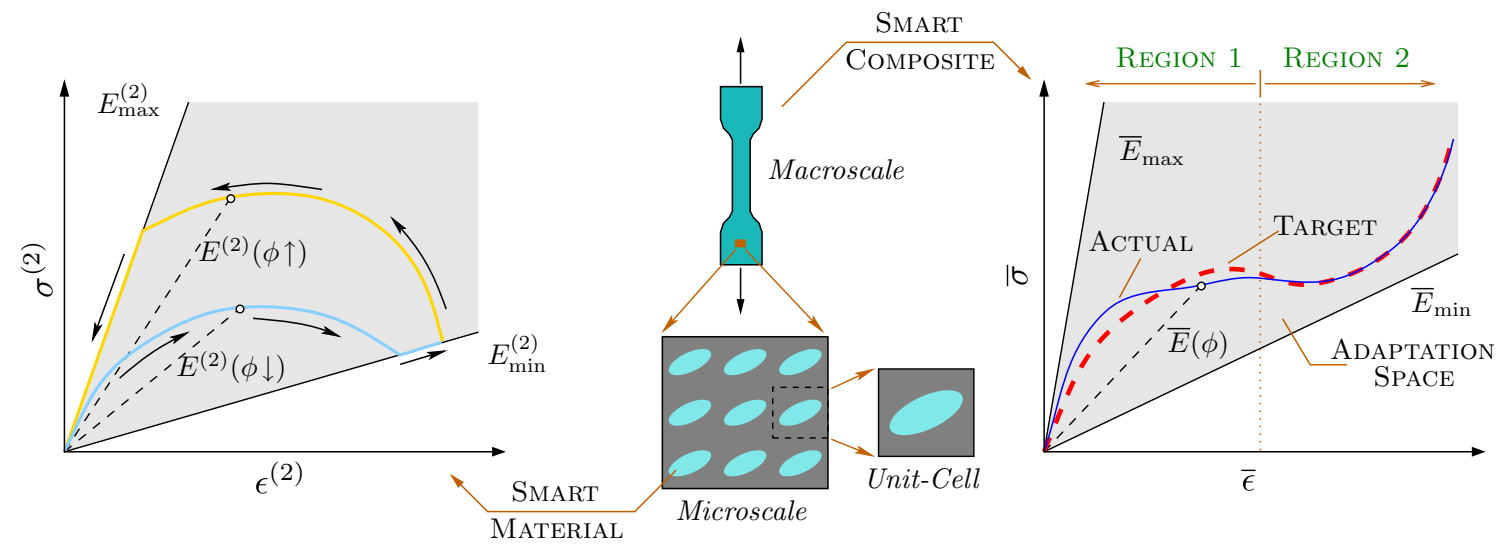

Fig. 2 Smart composite with a tunable stress-strain curve. The aim is to tune the elastic modulus $E^{(2)}(\phi)$ of a microscopic constituent (in this case the particle) via a control variable $\phi(t)$ so that the actual macroscopic stress $\bar{\sigma}(t)$ approaches a desired value $\bar{\sigma}^{*}(t)$ as quickly as

\subsection{Tunable mechanics}

Based on the simplified one-dimensional setting of Sect. 2.1.2, it will further be assumed that the first constituent has a fixed elastic modulus $\left(E^{(1)}\right)$ whereas the second constituent has a variable one $\left(E^{(2)}\right)$. Moreover, suppose that the second constituent has a control variable $\phi$, such as the magnetic field in the case of magnetorheological elastomers, so that the value of $E^{(2)}$ can be varied between minimum and maximum values:

$E_{\min }^{(2)} \leq E^{(2)}(\phi) \leq E_{\max }^{(2)}$

In practice, the control variable is a function of time. During the development of the control framework of this work, the particular form of the signal $\phi(t)$ and the functional form of $E^{(2)}(\phi)$ will actually not be relevant-the temporal variation of $E^{(2)}$ will be directly controlled instead. Presently, however, in order to demonstrate the control idea, it will be assumed that $E^{(2)}$ is a non-decreasing function of $\phi$. The macroscopic elastic modulus, therefore, also becomes a (nondecreasing) function of $\phi$. Now, if $\phi$ is varied together with a prescribed strain signal $\epsilon^{(2)}(t)$, the microscopic stress-strain response $\sigma^{(2)}(t)=E^{(2)}(\phi) \epsilon^{(2)}(t)$ of the second constituent can follow a highly nonlinear curve. Consequently, the macroscopic response $\bar{\sigma}(t)=\bar{E}(\phi) \bar{\epsilon}(t)$ of the composite can also be highly nonlinear so that, by properly adjusting the variation of $\phi(t)$, the actual stress signal $\bar{\sigma}(t)$ can be controlled in order to follow a target signal $\bar{\sigma}^{*}(t)$. Within this framework, $\bar{\epsilon}(t)$ is prescribed, $\phi$ (or, eventually directly $\left.E^{(2)}\right)$ is the input that is controlled and $\bar{\sigma}$ is the output that helps assess the control error.

These ideas which underlie tunable mechanics at the microscopic and macroscopic scales are demonstrated in possible and tracks this target signal with high accuracy. A numerical example which closely follows this problem depiction will be presented in Sect. 4.2.1

Fig. 2 for a generic periodic microstructure. The degree of accuracy with which $\bar{\sigma}$ follows $\bar{\sigma}^{*}$ depends on the controller as well as on the microstructure. In particular, the controller determines the speed with which $\bar{\sigma}$ captures $\bar{\sigma}^{*}$, typically displaying a transient part (Region 1 ) where the actual response rapidly approaches the target, followed by a steady-state part (Region 2) where a high accuracy is achieved. The microstructure, on the other hand, controls the degree of freedom in the macroscopic response (adaptation space) that is characterized by the maximum $\left(\bar{E}_{\max }\right)$ and minimum $\left(\bar{E}_{\text {min }}\right)$ elastic moduli. It is desirable to choose or design the microstructure so that the adaptation space contains the target signal at all times. These aspects will be further discussed in upcoming sections.

\subsection{Templates for cyclic paths in stress-strain space}

\subsubsection{Macroscopic stress and strain signals}

Two simplifications will underlie the development of the control framework, based on the setup of Sect. 2.2 and along the goals stated in Sect. 1. First, instead of controlling $\bar{E}$ through an input $\phi(t), \bar{E}(t)$ will be controlled directly. In practice, materials change their mechanical response due to an external stimulus, for instance by controlling the temperature or the magnetic field, and the response of $\bar{E}$ to this stimulus is not immediate so that its incorporation requires additional modeling effort. Although this adds a layer of complexity to the control framework, the present aim is to address fundamental challenges that already exist under $\bar{E}(t)$-control. Second, it is clear that complex paths may be generated in the stress-space when $\bar{E}$ and $\bar{\epsilon}$ change simultaneously. Alternatively viewed, complex target paths that are defined by $\bar{\epsilon}(t)$ and $\bar{\sigma}^{*}(t)$ may be followed with an appropriate controller which tunes $\bar{E}(t)$. 
For the actuator-type applications which were mentioned in Sect. 1, both $\bar{\epsilon}(t)$ and $\bar{\sigma}^{*}(t)$ are cyclic signals. The phase of one signal with respect to the other together with their amplitudes, means and periods control the particular cyclic path in the stress-strain space. These degrees of freedom in the signals will be reduced without any impact on the control development by fixing the steady-state strain signal to a sinusoidal one with fixed mean $\left(\bar{\epsilon}_{o}\right)$, amplitude $(\Delta \bar{\epsilon} / 2)$ and $\operatorname{period}\left(T_{\epsilon}\right)$ :

$\bar{\epsilon}(t)=\bar{\epsilon}_{o}-\Delta \bar{\epsilon} \cos \left(2 \pi t / T_{\epsilon}\right)$.

The target steady-state stress signal, on the other hand, will have a variable mean $\left(\bar{\sigma}_{o}^{*}\right)$, amplitude $\left(\Delta \bar{\sigma}^{*} / 2\right)$, period $\left(T_{\sigma}\right)$ as well as a phase $(\theta)$ :
$\bar{\sigma}^{*}(t)=\bar{\sigma}_{o}^{*}-\Delta \bar{\sigma}^{*} \operatorname{cyc}\left(2 \pi t / T_{\sigma}+\theta\right)$.

Here, $c y c$ represents any cyclic signal, such as a sinusoidal or a triangular pattern. In practice, the strain as well as the target stress signals will be gradually increased towards the mean of these steady-state fluctuations through a short transition period-see Fig. 4. The particular choice for this transition does not influence the core aspects of the controller design and therefore will not be explicitly noted. Also note that changes in the mean or the amplitude of a signal lead to straightforward shifting or scaling along the corresponding axis in the stress-strain space and therefore will not be shown. In a multi-dimensional setting, all non-zero strain components will be assigned the same variation (2.5) while individual target stress signals may differ.

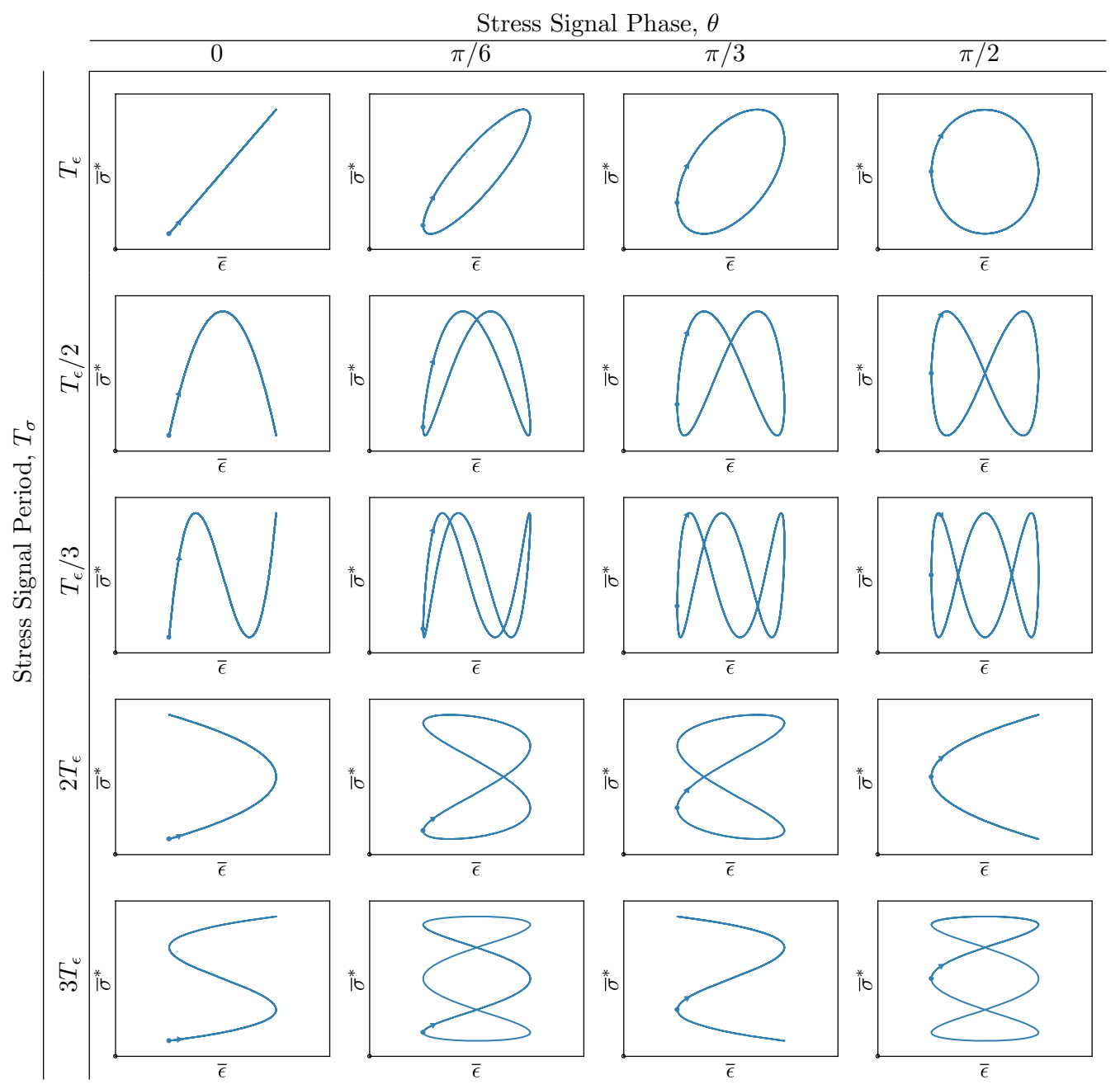

Fig. 3 The influence of the period mismatch $T_{\sigma} / T_{\epsilon}$ and the phase $\theta$ on the cyclic stress-strain path is summarized, using $c y c=\cos$ in (2.6). The period mismatch bends the initially straight path into a curved one while the phase splits the line into a closed path. The circle (o) at the origin indicates $\left(\bar{\epsilon}, \bar{\sigma}^{*}\right)=(0,0)$, the starting point along the cyclic path is indicated with a bullet $(\bullet)$ and the direction of motion is indicated with an arrow $(\triangleright)$. (Color figure online) 


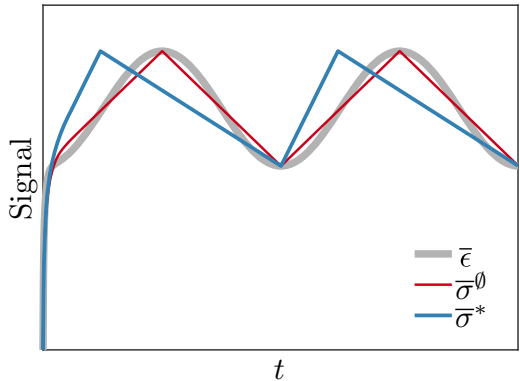

(a) Signal variations

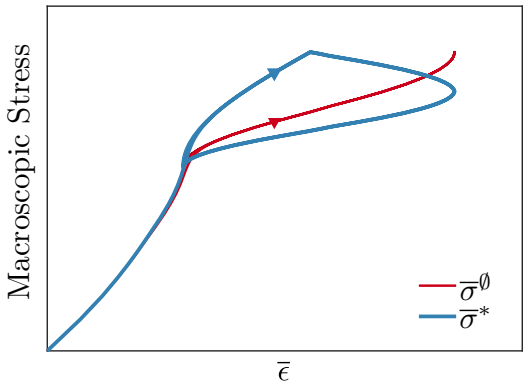

(b) Cyclic paths

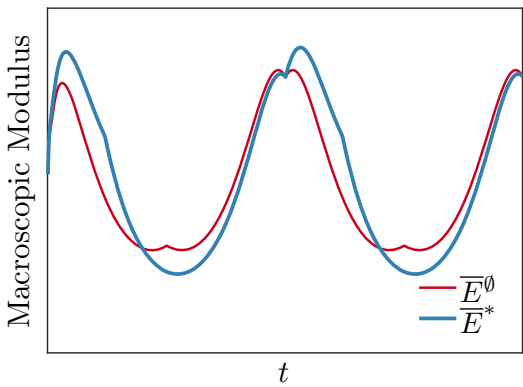

(c) Macroscopic moduli variations

Fig. 4 The influence of a triangular choice for $c y c$ in (2.6) is summarized, with $(\varnothing)$ and without $(*)$ matching peaks for the stress and strain signals. The transition part of the signals are also displayed. The default target macroscopic stress will be chosen as the $\bar{\sigma}^{*}(t)$ signal shown here

\subsubsection{Period ratio and phase}

The purpose of this section is to provide templates for complex cyclic paths in stress-strain space. The aim is to highlight that such paths can actually be easily achieved by, for instance, simple changes in the period ratio $T_{\sigma} / T_{\epsilon}$ and the phase $\theta$. Both of these parameters have central practical roles. For instance, achieving damping in cyclic motion requires a phase between the stress and strain signals. The period of the two signals also do not need to match. As an extreme case, for instance, one may wish to keep the macroscopic load (or, stress) at a constant value through cyclic macroscopic deformation (or, strain). In another extreme case, the motion of an object cannot be initiated if frictional resistance is not overcome despite cyclic macroscopic loading. In the former case, the material in the actuator should be able to follow $T_{\sigma} / T_{\epsilon} \rightarrow \infty$ whereas in the latter case $T_{\sigma} / T_{\epsilon} \rightarrow 0$. Clearly, any other ratio between these two extremes is also conceivable.

Figure 3 summarizes the influence of these parameters when $c y c=\cos$ is chosen for the stress signal (2.6). Here, only the steady-state paths are shown. When $T_{\sigma}=T_{\epsilon}$ and $\theta=0$, the cyclic path follows a straight line. Even then, however, this line cannot be followed with an elastic material with a constant $\bar{E}^{*}=\bar{\sigma}^{*} / \bar{\epsilon}$ because it does not extrapolate down to the origin due to the particular choices for $\bar{\epsilon}_{o}$ and $\bar{\sigma}_{o}^{*}$, so that tunable mechanics would be required already. When a period mismatch is added without phase, the straight path is bent into a curved one, displaying an increasing number of inflection points with an increasing mismatch. On the other hand, when a phase $\theta>0$ is added at matching period, the straight line is split open towards a closed cyclic path, with the direction of motion being clockwise. When $\theta^{\prime}=\theta+\pi / 2$ is added as the phase, the cyclic path flips upside down, but the direction of motion is retained. When $\theta^{\prime}=\theta+\pi$ is added, both the path flips upside down and the direction of motion is reversed. Hence, only $\theta \leq \pi / 2$ is shown.

\subsubsection{Signal shape}

The shape of the stress signal is an additional parameter. Figure 4 displays the influence of a triangular choice for $c y c$ in (2.6). Here, two specific choices are displayed, both with $T_{\sigma}=T_{\epsilon}$. In the first one, the peaks of the stress and strain signals match, which qualitatively corresponds to zero phase (indicated by $\emptyset$ ). Because there is no phase, the path in the stress-strain space is not split. However, it is wavy rather than straight due to the non-matching shapes of the two signals. In the second choice, the peak of the triangular stress signal is shifted, which effectively introduces a phase and hence splits the cyclic path. This signal, together with its transition part, will be chosen as the default target macroscopic stress variation $\bar{\sigma}^{*}(t)$ in the SISO setting. Note that the difference between the macroscopic modulus variations for the two choices discussed are seemingly small, yet the impact of this difference on the stress-strain paths is significant. This highlights the need for a tuning approach that directly assesses the error in the stress rather than the modulus. The design of an appropriate controller will be discussed in Sect. 3.1. Before this discussion, the ability to tune the macroscopic mechanics and typical performance indicators will be discussed. This discussion will be cast in a series of examples which highlight the micromechanical aspects that influence the tuning ability.

In all of these examples, the base controller of Sect. 3.1 is employed, which specifically makes use of the fact that a cyclic (or, periodic) path is being targeted. Moreover, the default macroscopic signals are assigned $\left\{\bar{\epsilon}_{o}, \Delta \bar{\epsilon}, T_{\epsilon}\right\}=$ $\{0.02,0.01,5 \mathrm{sec}\}$ and $\left\{\bar{\sigma}_{o}, \Delta \bar{\sigma}\right\}=\{1.05 \mathrm{MPa}, 0.25 \mathrm{MPa}\}$. Finally, unless otherwise noted, $E^{(1)}=50 \mathrm{MPa}$ and $f^{(1)}=$ $f^{(2)}=0.5$. Note that the particular value of $T_{\epsilon}$ will not be important-it can be increased/decreased arbitrarily to describe low/high frequency phenomena. For this reason, the variation of control quantities will be monitored with respect to the number of cycles, instead of with respect to time. Similarly, when a parameter which involves the unit of time appears, its magnitude should be judged relative to $T_{\epsilon}$. 


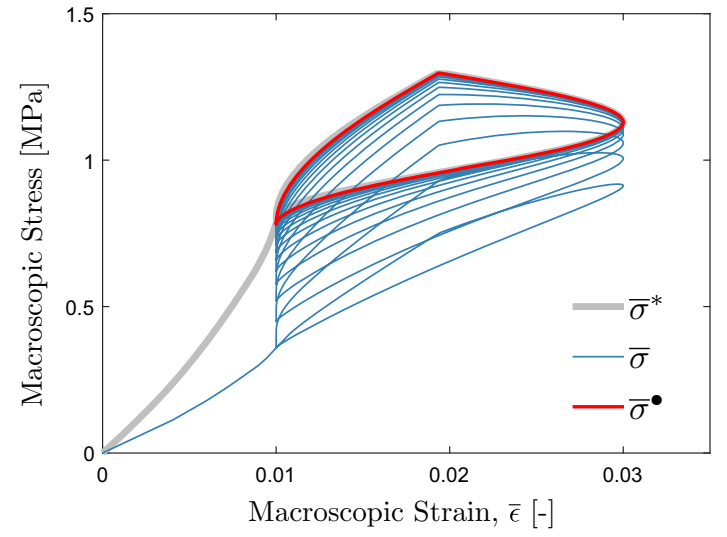

(a) Macroscopic path

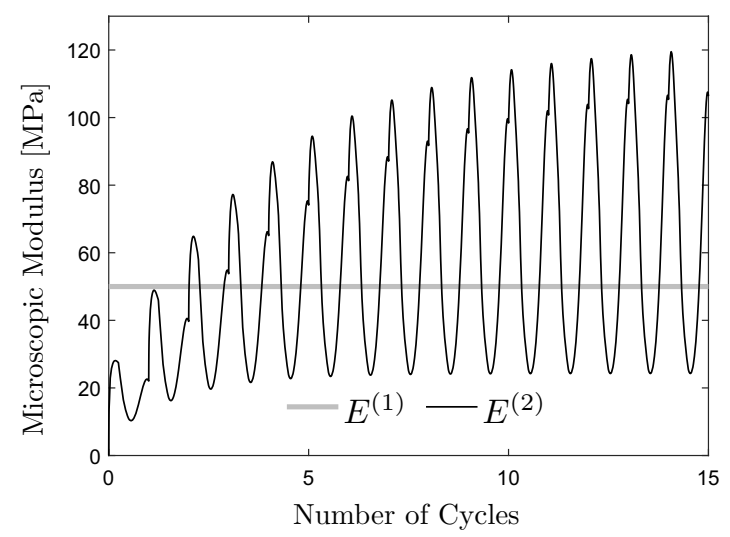

(c) Microscopic modulus

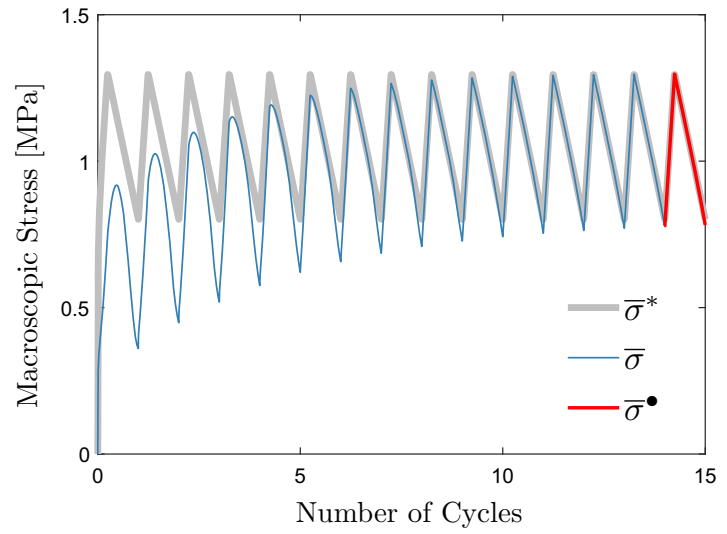

(b) Macroscopic stress

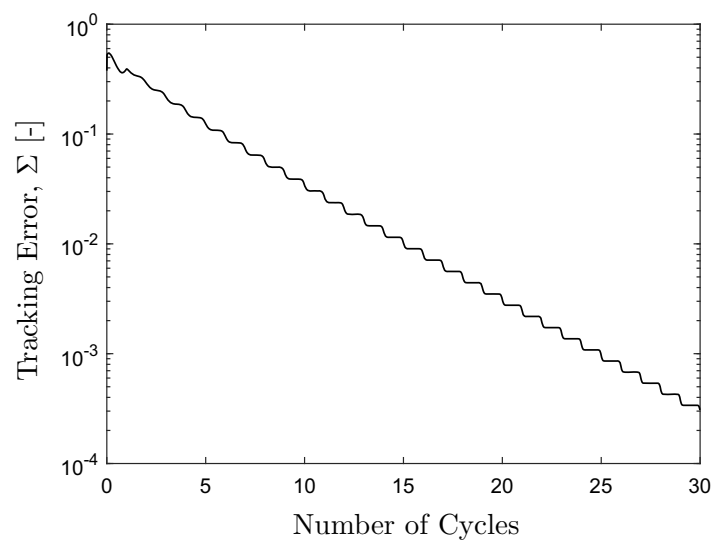

(d) Tracking error

Fig. 5 The controller performance is demonstrated for the macroscopic modulus model $\bar{E}_{\|}$from (2.3) 1 . The tracking error from (2.7) decreases below one percent after 15 cycles. The $\bar{\sigma}$ signal over the last cycle and its path in the macroscopic stress-strain space is indicated with the $\bar{\sigma}^{\bullet}$ curve

\subsection{Base controller performance}

\subsubsection{Elastic model with linear control}

Among the macroscopic moduli of (2.3), the model based on $\bar{E}_{\|}$is linear in the tunable microscopic modulus $E^{(2)}$ whereas $\bar{E}_{\perp}$ is nonlinear. In a first step, the linear model will be employed in order to predict the macroscopic response of the composite via $\bar{\sigma}=\bar{E}_{\|} \bar{\epsilon}$. In order to assess the controller performance, the tracking error $(\Sigma)$ is defined by evaluating the error in the immediate past over a duration of one period:

$\Sigma(t)=\left(\frac{1}{T_{\sigma}} \int_{t-T_{\sigma}}^{t}\left(\frac{\bar{\sigma}-\bar{\sigma}^{*}}{\bar{\sigma}^{*}}\right)^{2} \mathrm{~d} t\right)^{1 / 2}$

For $t<T_{\sigma}$, the duration of averaging is limited to the history. In a multi-dimensional setting, the notation $\Sigma_{i j}$ will be employed to refer to the particular stress component $\bar{\sigma}_{i j}$ for which the error is calculated with respect to a target signal $\bar{\sigma}_{i j}^{*}$.
Figure 5 summarizes the output of the base controller for this setting. Clearly, the chosen controller type can drive the macroscopic response towards the target signal. In about 15 cycles, the tracking error $\Sigma$ already indicates less than one percent deviation from the target signal. This last cycle will be explicitly shown in the macroscopic stress-strain space in order to highlight its excellent visual agreement with the target signal. In order to achieve this output, the tunable microscopic modulus $E^{(2)}$ varies significantly, but within the same order of magnitude as $E^{(1)}$. This indicates that mediocre tunability may in practice be sufficient to track complex cyclic paths. Note that the initial value of $E^{(2)}$ does not have a significant impact on the tracking error variation and hence is taken to be an arbitrarily small value by default in all examples.

A number of practical issues may arise during control, one of which is demonstrated in Fig. 6. In practice, as previously discussed in Sect. 2.2 and depicted in Fig. 2, there may be limits to the range over which $E^{(2)}$ may be varied. In comparison to Fig. 5, if $E^{(1)}$ is decreased to $35 \mathrm{MPa}$ from the default value of $50 \mathrm{MPa}, E^{(2)}$ must now achieve higher values 

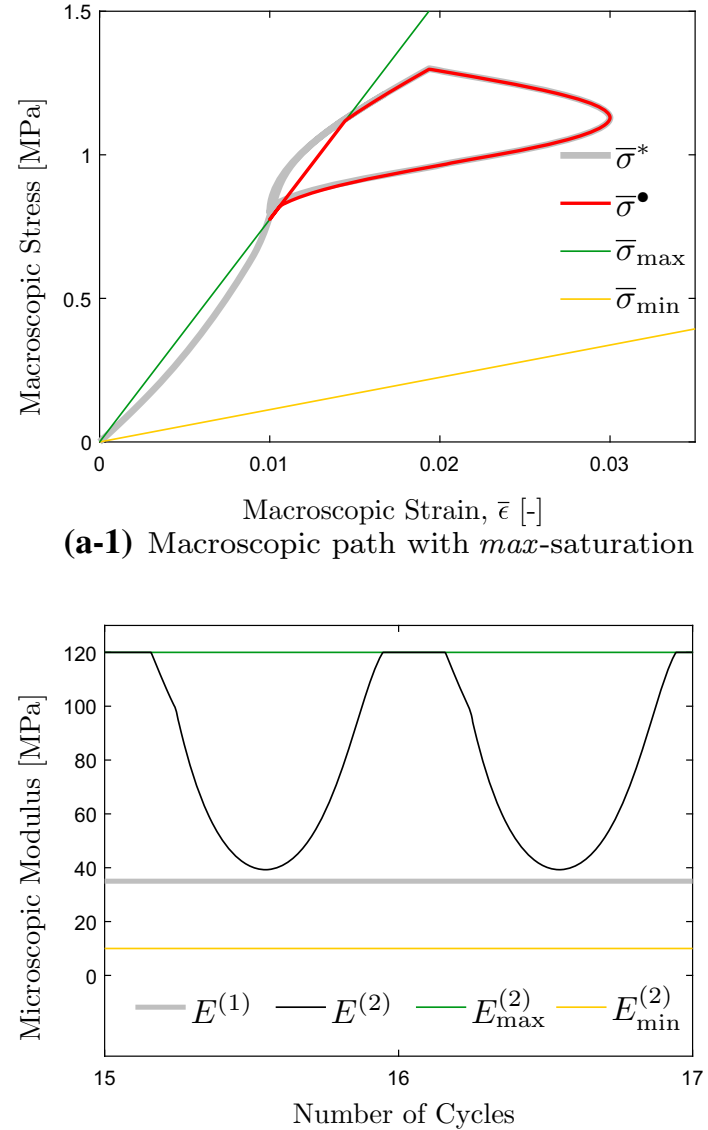

(a-2) Microscopic modulus with $\max$-saturation

Fig. 6 For the setting of Fig. 5, $E^{(1)}$ is varied in order to force $E^{(2)}$ towards imposed saturation limits $E_{\max }^{(2)}=120 \mathrm{MPa}$ and $E_{\min }^{(2)}=$ $10 \mathrm{MPa}$. For case (a), $E^{(1)}=35 \mathrm{MPa}$ for which $\bar{E}_{\max }=77.5 \mathrm{MPa}$

over a cycle. If, however, $E_{\max }^{(2)}=120 \mathrm{MPa}$ is enforced then $E^{(2)}$ saturates at this value over portions of the cycle, effectively limiting $\bar{E}$ to a maximum value $\bar{E}_{\max }$ and, thereby, $\bar{\sigma}$ to $\bar{\sigma}_{\max }=\bar{E}_{\max } \bar{\epsilon}$. This $\max$-saturation reflects as a constantmodulus response, i.e. a line which may be extrapolated to the origin, in the corresponding portion of the cyclic path in the macroscopic stress-strain space. A similar min-saturation effect may also be observed, for instance if $E^{(1)}=75 \mathrm{MPa}$ is employed and $E^{(2)}$ is limited to $E_{\min }^{(2)}=10 \mathrm{MPa}$, which limits $\bar{\sigma}$ to $\bar{\sigma}_{\min }=\bar{E}_{\min } \bar{\epsilon}$. In such cases, the tracking error over a period will remain at a relatively large value although it is observed that the pointwise error in portions of the cyclic path where saturation does not occur is negligible. In practice, referring to Fig. 2, determination of the bounds for the adaptation space may be considered as a preprocessing stage where the saturation values of the tunable microscopic constituent(s) are checked. Subsequently, the target signal should either be chosen to lie within this space or the significance of the error due to saturation must be assessed.
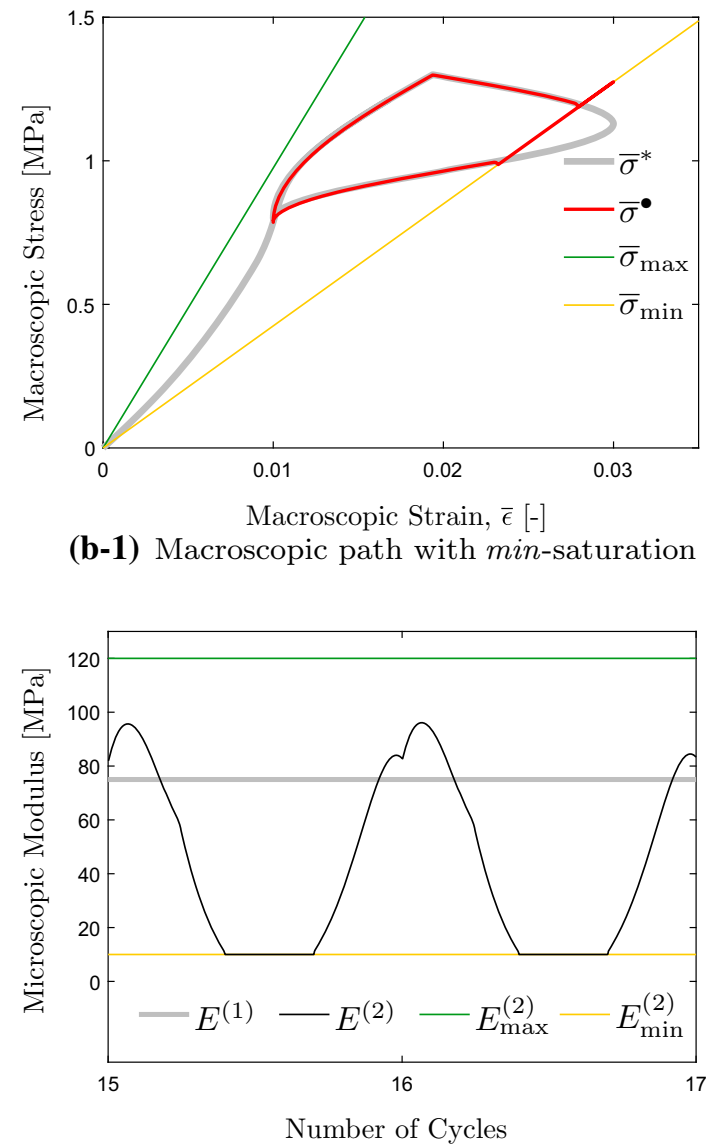

(b-2) Microscopic modulus with min-saturation

and $\bar{E}_{\min }=22.5 \mathrm{MPa}$, leading to max-saturation. For case (b), $E^{(1)}=$ $75 \mathrm{MPa}$ for which $\bar{E}_{\max }=97.5 \mathrm{MPa}$ and $\bar{E}_{\min }=42.5 \mathrm{MPa}$, leading to min-saturation. The macroscopic stress $\bar{\sigma}$ saturates to either $\bar{\sigma}_{\max }=\bar{E}_{\max } \bar{\epsilon}$ or $\bar{\sigma}_{\min }=\bar{E}_{\min } \bar{\epsilon}$ type response

\subsubsection{Control approach advantages}

Clearly, in the particular setting of the previous section, one may easily calculate the value of $E^{(2)}$ via $(2.3)_{1}$ so that $\bar{E}$ matches the desired value $\bar{E}^{*}=\bar{\sigma}^{*} / \bar{\epsilon}$ without the need for a control approach. It is therefore important at this stage to digress momentarily from the numerical investigations and emphasize two outstanding advantages of the control approach over such an alternative, in order to also shed light on the developments of the following sections:

1. Computational complexity As already commented in Sect. 2.1.1, the inverse problem of determining the optimal microscopic moduli for a desired macroscopic one is not straightforward in a multi-dimensional setting. Although this is not a challenging task, it is significantly costly because it will require solving multiple cell problems of homogenization at each step of an iterative optimization problem. Subsequently, this task needs to be 
repeated at each time step along the macroscopic stressstrain path. The control approach, on the other hand, will always carry out a single cell-level computation for a time step in order to determine the microscopic stress field, which delivers the macroscopic stress through cellaveraging.

2. Microscopic uncertainty In the context of an inverse problem, the characterization of the macroscopic response towards a macroscopic modulus rests on assumptions which can be easily violated in practice, for instance: (i) the microscopic mechanical response is purely elastic, (ii) the microscopic elastic moduli are known precisely, (iii) the microstructure topology is known precisely. Uncertainties in the microscopic mechanical behavior or properties as well as a lack of precise knowledge of the microstructure will always introduce an error if one attempts to make a macroscopic response prediction through the computation of a macroscopic material property, in the one-dimensional case through $\bar{E}$. On the other hand, under appropriate feasibility conditions that will be further commented upon, the tunable microscopic constituent may always be controlled in order to direct the macroscopic stress signal towards the target variation.

To summarize, the control approach effectively considers the smart composite as a black box system which delivers a measurable stress for a given input signal, without a precise consideration of the microscopic details. Indeed, a microscopic computation in the present work serves precisely the purpose of measuring the response from a black box system. A future aim would be to replace computation with experiment such that the actual smart composite reacts to the control input, which is to be tuned until a desired steady-state output is achieved.

\subsubsection{Elastic model with nonlinear control}

The particular microstructure topology employed has a significant impact on control capability. In order to demonstrate this aspect, the macroscopic modulus model $\bar{E}_{\perp}$ of $(2.3)_{2}$ will be employed with the default numerical parameters, with the exception of $E^{(1)}=15 \mathrm{MPa}$. For clarity, no saturation limit is imposed on $E^{(2)}$. The results in Fig. 7 indicate that the control algorithm attempts to drive $E^{(2)}$ to ever larger values, although the tracking error saturates. The reason for this saturation is clearly observed in the macroscopic stressstrain space: despite the lack of a $\max$-saturation on $E^{(2)}$, the microstructure imposes a max-saturation on $\bar{E}$ because the modulus model $\bar{E}_{\perp}$ which represents this microstructure is limited to a finite value even for $E^{(2)} \rightarrow \infty$. Presently, this finite value is too low so that the target path lies entirely outside the adaptation space and hence the controller can drive the output to the boundary of this space at most. Whenever the microstructure topology imposes constraints on the macroscopic response such that the tracking error cannot be driven to zero, the target path will be referred to as unrealizable. When $E^{(1)}$ is increased so that the adaptation space starts to encompass the target path, the tracking error saturation value decreases although the target is still unrealizable. Once the target path is entirely contained in the adaptation space the error can then be driven to zero (Fig. 8a). Note that in the present setting the control problem is nonlinear in $E^{(2)}$ and the controller is able to address this nonlinearity to minimize the tracking error.

\subsubsection{Inelastic model}

Among microscopic uncertainties, the possible inelastic response of the constituents causes a nonlinear macroscopic mechanical response. In order to further demonstrate the versatility of the base controller, it will be applied to the case when the tunable constituent is still elastic but the other is viscoelastic, thereby inducing a macroscopic viscoelastic response as well. For this purpose, the layered composite model is again employed with parallel loading so that $\bar{\sigma}=f^{(1)} \sigma^{(1)}+f^{(2)} \sigma^{(2)}$ in view of (2.1) together with the fact that the strain is a constant over both constituents. The tunable constituent delivers $\sigma^{(2)}=E^{(2)} \bar{\epsilon}$. The viscoelastic constituent is modeled with the standard linear solid so that $\sigma^{(1)}=\sigma_{e}^{(1)}+\sigma_{v}^{(1)}$ with $\sigma_{e}^{(1)}=E_{\infty}^{(1)} \bar{\epsilon}$ and $\sigma_{v}^{(2)}=E_{v}^{(1)}\left(\bar{\epsilon}-\epsilon_{v}\right)$. The rate of the microscopic viscous strain $\epsilon_{v}$ is governed by the equation $\tau \dot{\epsilon}_{v}+\epsilon_{v}=\bar{\epsilon}$ where $\tau$ is the relaxation time. Here, $E_{\infty}^{(1)}=100 \mathrm{MPa}$ and $E_{v}^{(1)}=10 \mathrm{MPa}$ will be employed. For the case when $\tau=1 \mathrm{sec}$, the controller performance is summarized in Fig. 9. Due to viscoelasticity, $\bar{\sigma}$ even takes negative values in the early stages of loading. Subsequently, however, the controller quickly drives the macroscopic response towards the target. Note that the macroscopic stress-strain path would already exhibit hysteresis even with a constant $E^{(2)}$ because the relaxation time $\tau$ is very close to $T_{\sigma}=5 \mathrm{sec}$. Hence, the controller is also working against this hysteresis in trying to achieve the target signal. In fact, the controller performance is only weakly influenced by $\tau$. Figure $8 b$ shows that the target path is effectively achieved in comparable times despite significant changes in $\tau$, and even when it is larger than $T_{\sigma}$.

The suite of problems discussed in this section have highlighted the mechanics aspects and physical challenges that are associated with the control of smart composites. Simultaneously, the versatility of the underlying base controller has been demonstrated. A compact discussion of this controller and its further development towards cases of practical interest will be presented next. 


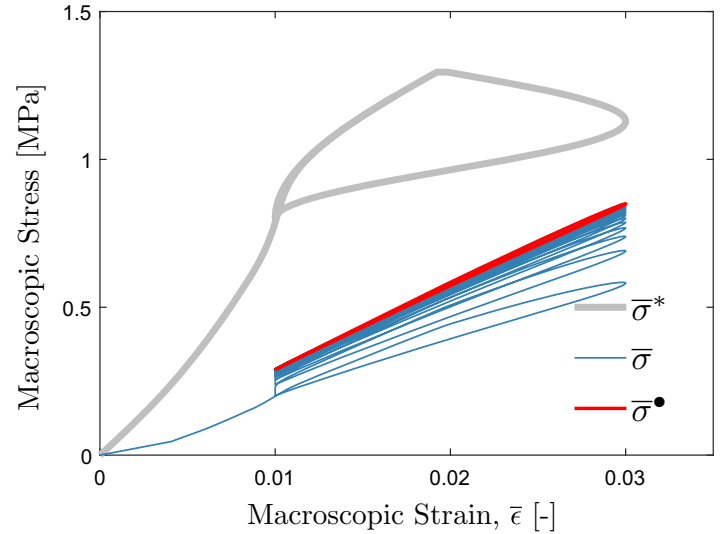

(a) Macroscopic path

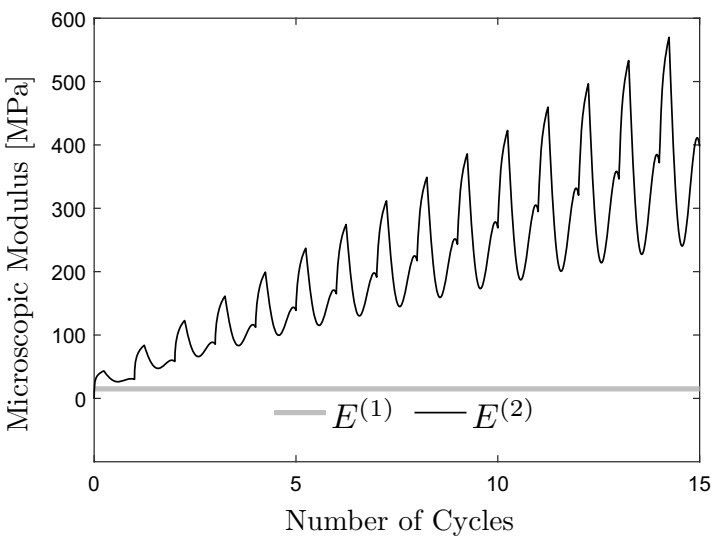

(c) Microscopic modulus

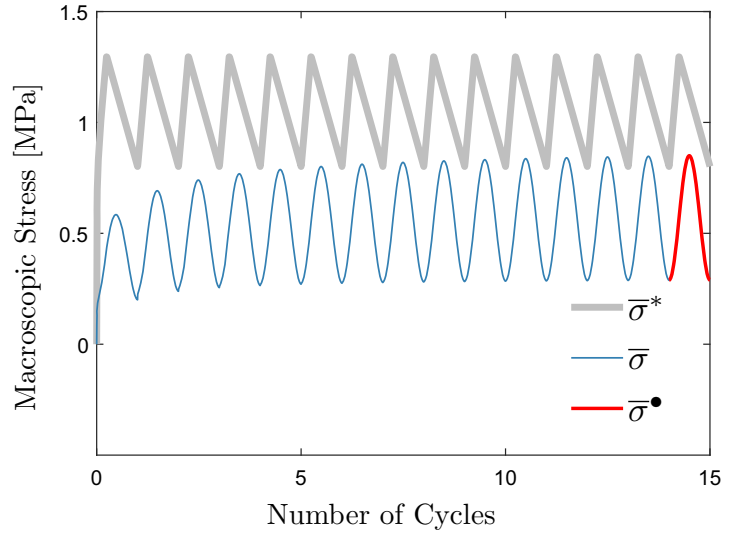

(b) Macroscopic stress

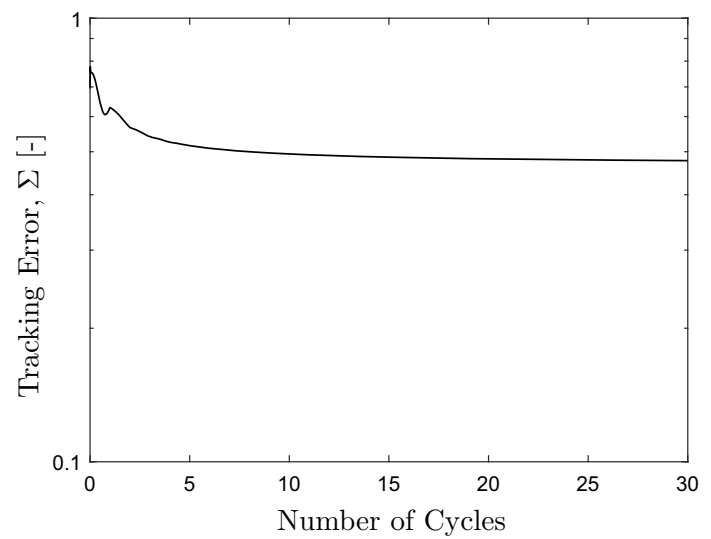

(d) Tracking error

Fig. 7 The controller performance is demonstrated for the macroscopic modulus model $\bar{E}_{\perp}$ from $(2.3)_{2}$. The target path is unrealizable due to the microstructure topology, leading to a saturating tracking error even if a continuous increase in $E^{(2)}$ is allowed

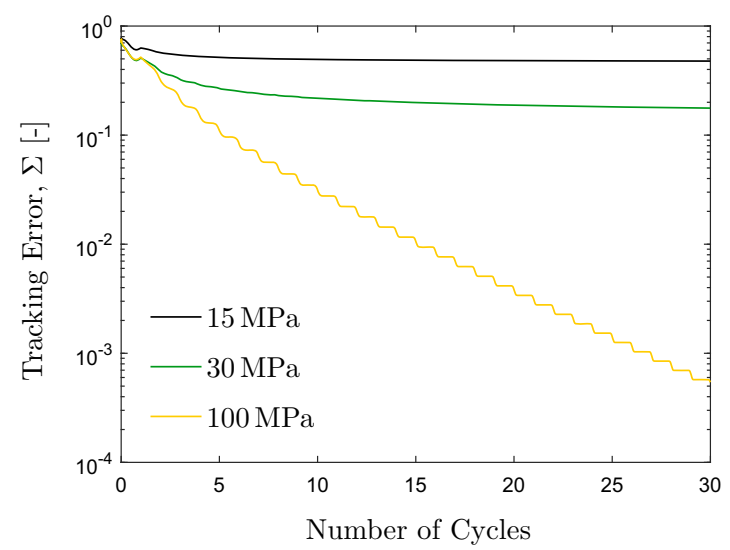

(a) Tracking error for different $E^{(1)}$

Fig. 8 Dependence of the tracking error on microscopic material properties: a $E^{(1)}$ is varied when the macroscopic response is described by $\bar{E}_{\perp}$ from $(2.3)_{2}$, eventually delivering a realizable response when $E^{(1)}$

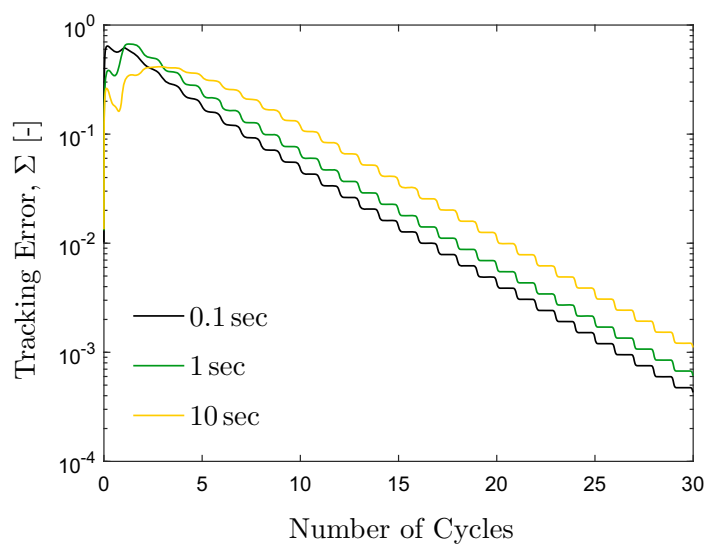

(b) Tracking error for different $\tau$

is sufficiently large, and $\mathbf{b}$ the relaxation time is varied beyond the period $T_{\sigma}=5 \mathrm{sec}$ for the case with a non-tunable viscoelastic constituent 


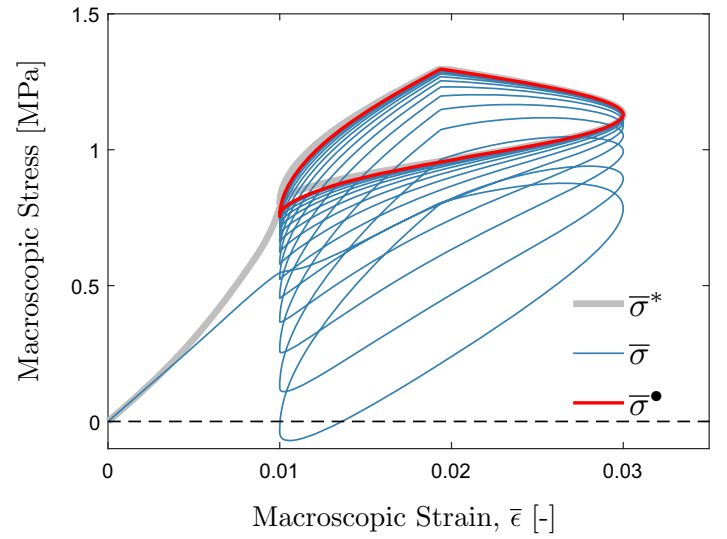

(a) Macroscopic path

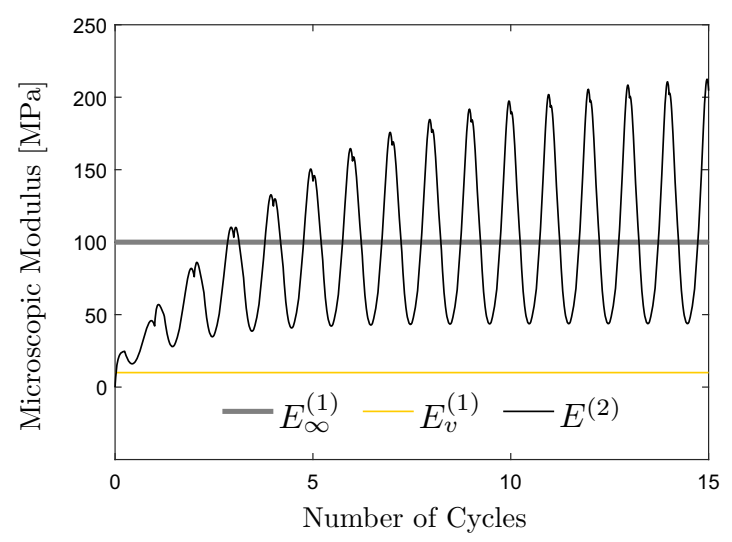

(c) Microscopic modulus

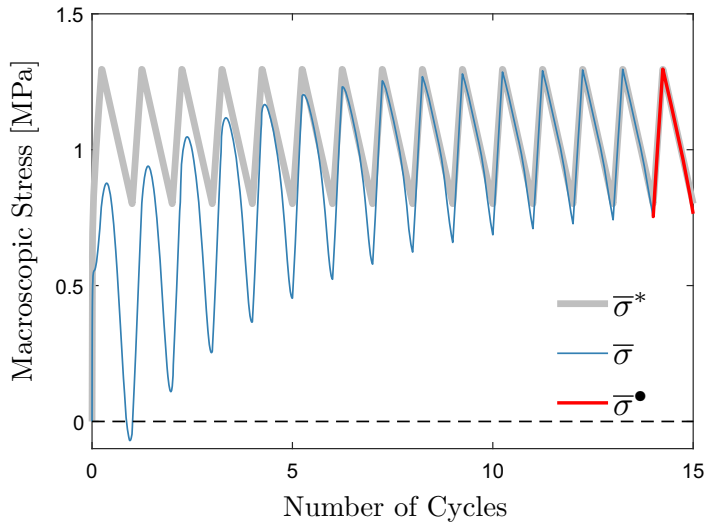

(b) Macroscopic stress

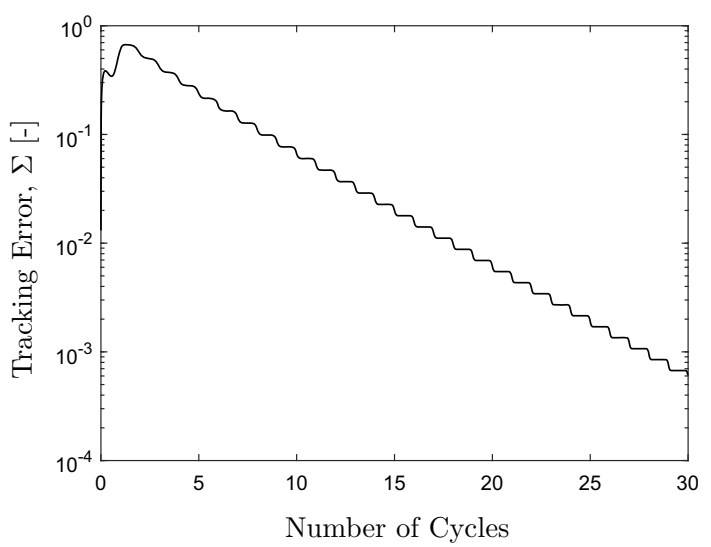

(d) Tracking error

Fig. 9 The controller performance is demonstrated for the case when the non-tunable constituent is viscoelastic, characterized by the material parameters $\left\{E_{\infty}^{(1)}, E_{v}^{(1)}, \tau\right\}$, with $\tau=1 \mathrm{sec}$

\section{Control framework}

In this section, the control framework related to the smart material system will be presented in a compact setting, with a specific focus on the non-standard aspects that have been adapted towards the purposes of this study. For background on the standard aspects of control theory, the reader is referred to [31-34]. The presentation will follow a SISO system configuration. However, the results of this section are generic enough to be used in a general Multi-InputMulti-Output (MIMO) system configuration that is needed for smart material control in a multi-dimensional framework, and this generalization will also be briefly commented upon. For further details regarding the design and operation of the underlying controller as well as its stability analysis, see [35].

\subsection{Repetitive controller}

A non-standard control algorithm that is suitable to the cyclic nature of the loading as well as for possible operation in a multi-dimensional setting is the repetitive controller, first introduced in [36]. The repetitive controller structure is devised based on the internal model principle, which states that a zero steady-state error controller with a specific structure can be designed for the system when the input characteristic is also repeated inside the controller [37]. Indeed, the base controller that was referred to earlier in Sect. 2.4 and which will be explicitly denoted in this section is built around this concept and consequently, as a specific example, the tracking error in the example of Fig. 5 already decreases below $10^{-7}$ after 70 cycles. Repetitive control is used for systems which have fixed periodic reference inputs. The practical applications of this approach to various problems are studied in [38]. The repetitive controller structure employed in this work is based on [39] and adapted towards the smart composite material system. The structure of this SISO controller is outlined in Fig. 10 and has the following features:

1. $C_{1}(s)$, the repetitive controller, minimizes the steadystate error of the control system. The controller reacts to the error $e(t)=\bar{\sigma}^{*}(t)-\bar{\sigma}(t)$. Because $e^{-\tau s}$ gives one 


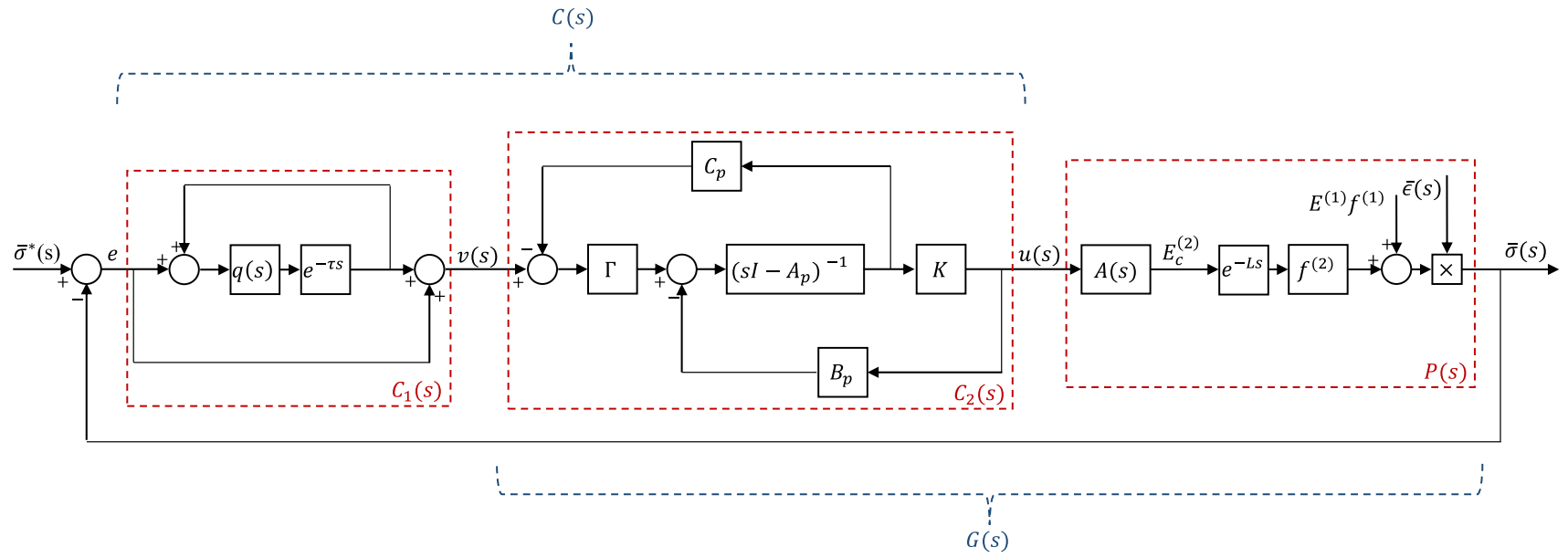

Fig. 10 The structure of the SISO controller in Laplace domain $(s)$

period delay $(\tau)$ to the error signal and feeds it to the current control calculations, the control scheme is endowed with a simple learning ability. Note that, in this context, the period $\tau$ corresponds to that which allows a cycle to be completed in the macroscopic stress-strain space, e.g. $\tau=\max \left\{T_{\epsilon}, T_{\sigma}\right\}$ if the period ratio (or its inverse) is an integer. Moreover, $q(s)$ is a proper low pass filter which serves two purposes. First, when the reference signal contains high frequency modes, for instance when it has a sharp corner, tracking may become unattainable. Second, the possible presence of delay in the system adversely impacts the stability of the system. One may address both of these problems by reducing the loop gain of the controller in the high frequency range, which is achieved by $q(s)$.

2. $C_{2}(s)$, the compensator, supports the repetitive controller by improving the transient response of the system. This controller is structured in state-space form, where optimal controllers can be designed directly, circumventing the need for explicit calibration of controller parameters. Moreover, this approach handles MIMO systems easily, thus enabling control in a multi-dimensional setting. Here, $A_{p}$ represents the system internal dynamics, $B_{p}$ is the input gain and the system output is calculated using $C_{p}$. Note that these may not be available explicitly but can be calculated numerically. Moreover, $\Gamma$ represents the Kalman filter gain and $K$ is the gain for the full-state feedback controller. Overall control, $C(s)$, is achieved through the combined action of the repetitive controller and the compensator.

3. $P(s)$, the plant, represents the physical system that the controller acts on. The smart composite response is represented here through the linear form $(2.3)_{1}$, where the controlled microscopic elastic modulus has been explicitly denoted with a subscript $(\cdot)_{c}$ for clarity. Note that any nonlinear relation, in particular $(2.3)_{2}$, can also be represented in a similar form after linearization for control purposes. The remaining ingredients are associated with physical actuation effects that are expected in an experimental setting: (i) an actuator delay of $L$ seconds is indicated externally through $e^{-L s}$, for instance due to the communication between the controller and the FEM simulation in Sect. 4, and (ii) $A(s)$ represents actuator dynamics such as inertia and will be taken in the form of a low pass filter. Controller stability relies on the combined structure of the plant and the compensator, referred to as the compensated plant, $G(s)$ —see [35] for relevant stability analysis.

Now, the base controller of Sect. 2.4 refers to a specialization of this controller structure, due to a simplified compensator structure with $C_{2}=K$ as well as due to the omission of the actuator dynamics $(A(s)=1)$, the delay $(L=0)$ and the filter $(q(s)=1)$. All upcoming examples, on the other hand, will assume the presence of these components. As a consequence, the tracking error will not steadily decay towards zero but will rather saturate at a small value.

\subsection{Multi-input-multi-output setting}

Control must be carried out in a MIMO setting in the multi-dimensional case where multiple stress signals must be tracked as the smart composite is subjected to multiple strain signals. As the details of the extension from SISO to MIMO depend on the particular case, the purpose of this section is to demonstrate the performance of the MIMO controller with a specific example without going into details of its structure, which basically entails the replacement of various scalars in Fig. 10 with vectors and matrices-see [35] for details. Specifically, the layered composite model from Sect. 2.1.2 will be employed in a biaxial loading scenario so 
Fig. 11 Controller design for the layered composite model under biaxial loading. The controller matrix $\boldsymbol{C}$ is based on Fig. 10 and hence is not explicitly depicted. Moreover, the volume fractions associated with the (linearized) macroscopic moduli are denoted through the matrix $\boldsymbol{F}$

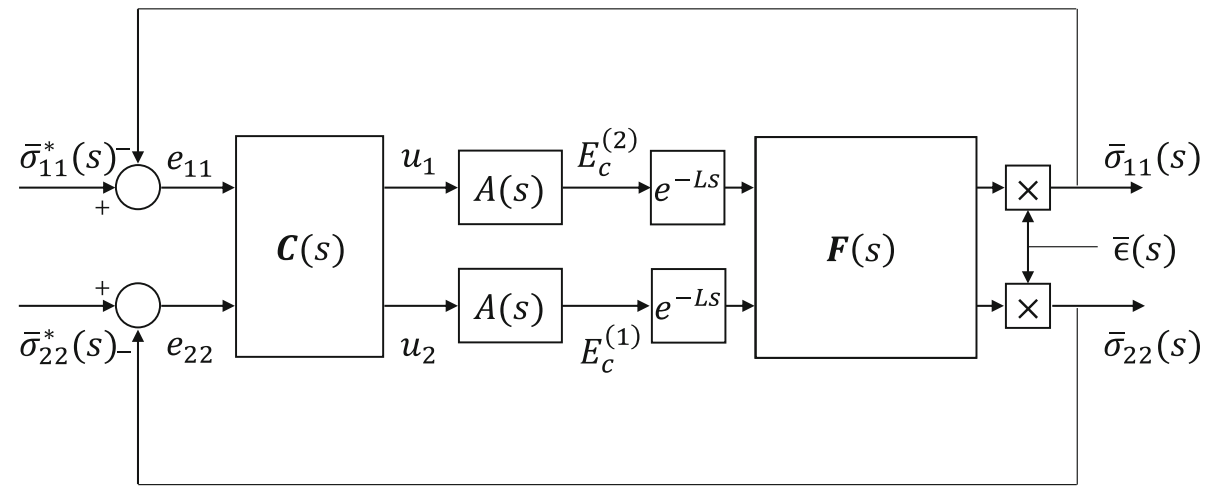

that the macroscopic response of the microstructure is available in closed-form. It is assumed that each constituent has a variable Young's modulus $E^{(I)}$ and zero Poisson's ratio. In addition, the strain function is assumed to be the same along both axes, $\bar{\epsilon}_{11}(t)=\bar{\epsilon}_{22}(t)=\bar{\epsilon}(t)$. The structure of the corresponding MIMO controller is outlined in Fig. 11. The results in Fig. 12 indicate that the performance of the MIMO controller is comparable to that of the SISO setting which was demonstrated in Sect. 2.4, with respect to both macroscopic target signals $\left(\bar{\sigma}_{11}^{*}(t)\right.$ and $\left.\bar{\sigma}_{22}^{*}(t)\right)$ which differ from each other in phase and shape. Specifically, the tracking error for both stress components rapidly approach their individual target signals which clearly lie within the adaptation space. It is important to highlight that this example already demonstrates the advantage of employing tunable microstructures as opposed to a fixed microstructure or a single homogeneous material with variable elastic properties: the former alternative is unable to adapt to the continuously changing macroscopic performance demands whereas the latter one is unable to provide independent adaptation for each target signal. Only a suitably chosen microstructure with tunable constituents is able to meet the demands of complex loading scenarios in order to ensure that the adaptation space is properly constructed.

It is instructive to discuss a simple generalization of the present example to further emphasize the impact of the microstructure on the adaptation space. If the layered composite is subjected to a shear strain $\bar{\epsilon}_{12}(t)$ in addition to the two normal strains with an accompanying target signal $\bar{\sigma}_{12}^{*}(t)$ then the macroscopic normal stress-strain relations $\bar{\sigma}_{11}=\bar{E}_{\|} \bar{\epsilon}_{11}$ and $\bar{\sigma}_{22}=\bar{E}_{\perp} \bar{\epsilon}_{22}$ should be augmented by the shear relation $\bar{\sigma}_{12}=2 \bar{\mu}_{\bar{\epsilon}}$. The macroscopic shear modulus $\bar{\mu}$ for such a composite follows the nonlinear expression $(2.3)_{2}$ in terms of the microscopic shear moduli $\bar{\mu}=\left(f^{(1)} / \mu^{(1)}+f^{(2)} / \mu^{(2)}\right)^{-1}$. Now, it appears that both the Young's and shear moduli of the microscopic constituents are available for tuning. However, additionally recalling the particular choice of a zero Poisson's ratio in this example, there holds $\mu^{(I)}=E^{(I)} / 2$ so that one simply obtains a similar relation $\bar{\mu}=\bar{E}_{\perp} / 2$ for the composite response, leading to the expression $\bar{\sigma}_{12}=\bar{E}_{\perp} \bar{\epsilon}_{12}$. Consequently, only one of the two signals $\bar{\sigma}_{22}$ and $\bar{\sigma}_{12}$ may be ensured to track its target by controlling $\bar{E}_{\perp}$, which will dictate the variation of the remaining signal. Hence, it is clear that the microstructure topology can easily inhibit tunability of the composite, rendering the overall target unrealizable. A simple layered microstructure is conceptually limited from the outset for addressing the present problem because there are only two control variables $E^{(I)}$ for three target signals. Therefore, an immediate remedy for the present example is to incorporate a third tunable layer into the microstructure. However, the critical role of the microstructure topology persists. Clearly, irrespective of the number of tunable constituents, there will always be only two microscopic control variables effectively in action as long as the microstructure is assigned a simple layered structure. This highlights the need for more complex distributions of the tunable constituents. The design of the microstructure so as to ensure independent adaptation for arbitrary loading scenarios with multiple target signals in a multi-dimensional setting is an outstanding issue that will not be addressed presently.

\section{FEM-based simulations}

\subsection{Numerical setup}

In a multi-dimensional setting, the macroscopic stress field is available in closed-form in only a few exceptional cases, one of which was discussed in Sect. 3.2. In order to demonstrate the versatility of the overall control framework towards tunable mechanics for smart materials with arbitrary microstructures, the approach developed so far will now be integrated with a FEM-based computation environment. Consequently, the solution of a single boundary value problem will be required at each time step. Although this leads to a significant rise in computation time compared to earlier examples, the examples to be discussed will demonstrate that the framework remains feasible for both twoand three-dimensional microstructures. In all cases, peri- 


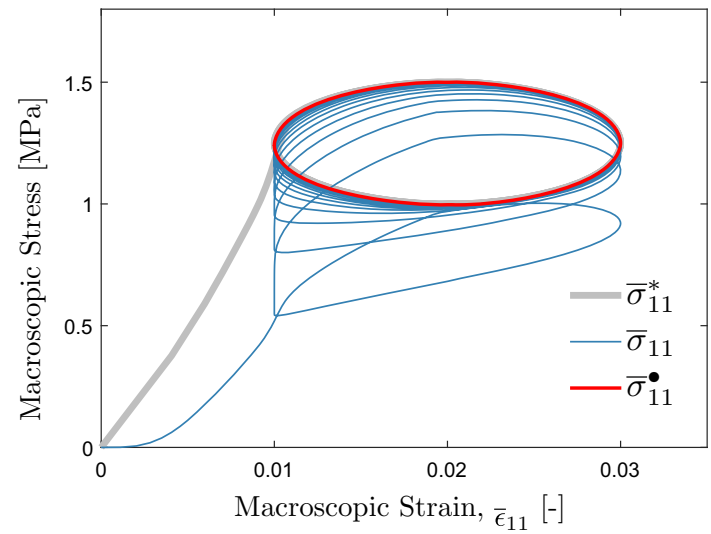

(a) Macroscopic path

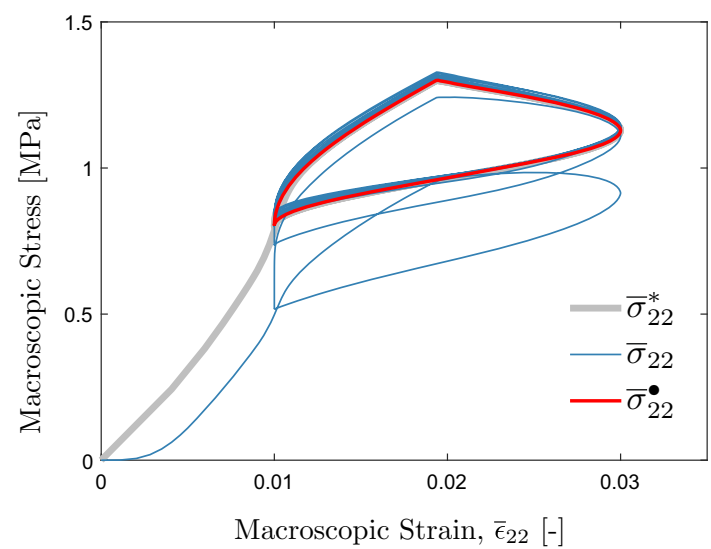

(c) Macroscopic path

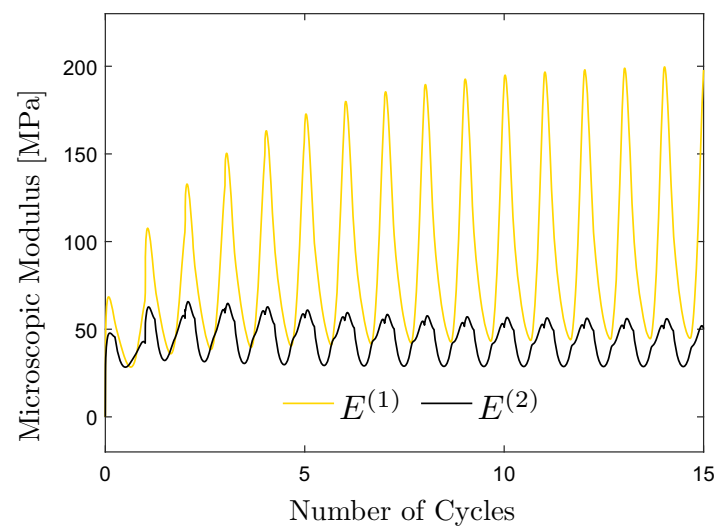

(e) Microscopic modulus

Fig. 12 The controller performance is demonstrated for the layered composite of Sect. 3.2. Here, $\bar{\sigma}_{11}^{*}$ is constructed using $c y c=\cos$ with $T_{\sigma}=T_{\epsilon}$ and $\theta=\pi / 2$ (see Fig. 3) as well as $\left\{\bar{\sigma}_{o}, \Delta \bar{\sigma}\right\}=$ $\{1.25 \mathrm{MPa}, 0.25 \mathrm{MPa}\}$ in (2.6) whereas $\bar{\sigma}_{22}^{*}$ is based on the default signal

odic microstructures are discussed so that the macroscopic strain is imposed through periodic boundary conditions on the unit-cell. The non-zero macroscopic strain components

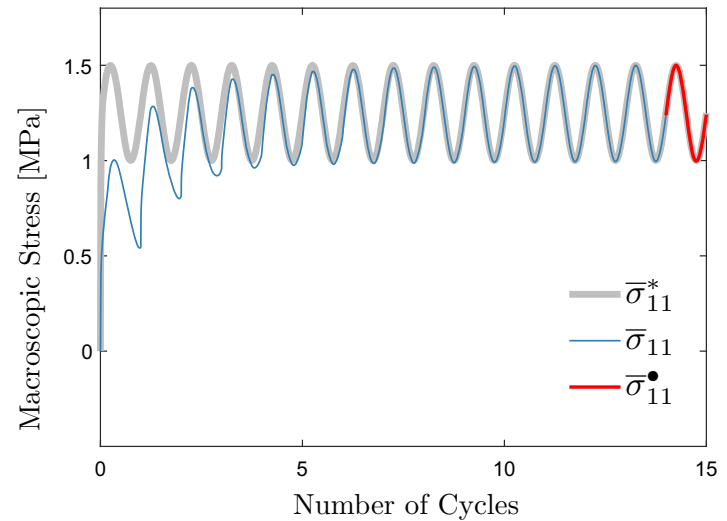

(b) Macroscopic stress

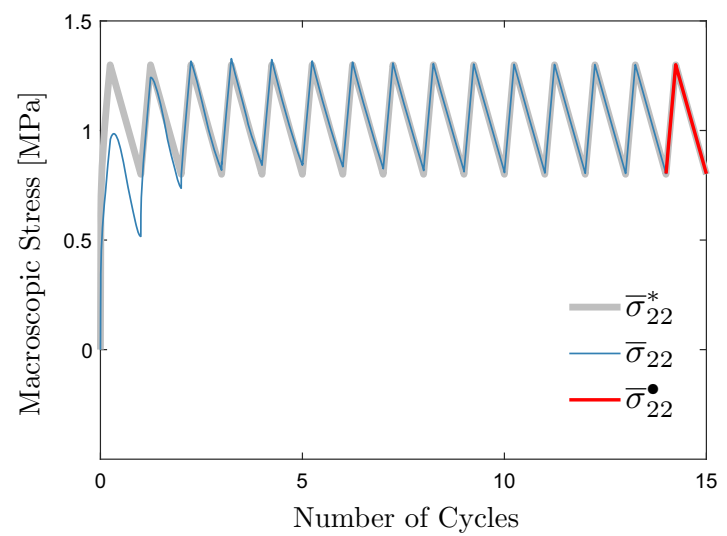

(d) Macroscopic stress

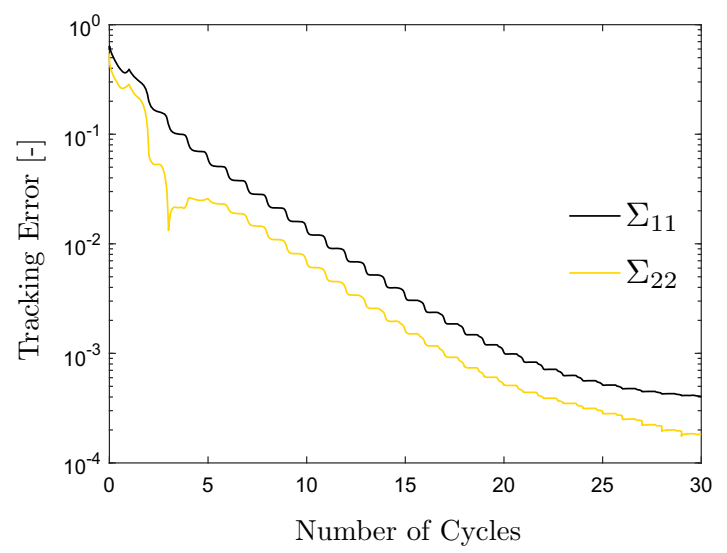

(f) Tracking error

from Fig. 4. All non-zero strain components (presently $\left\{\bar{\epsilon}_{11}, \bar{\epsilon}_{22}\right\}$ ) have the same variation (2.5) in all multi-dimensional examples, as noted in Sect. 2.3.1

will again be assigned the signal described by $\left\{\bar{\epsilon}_{o}, \Delta \bar{\epsilon}, T_{\epsilon}\right\}=$ $\{0.02,0.01,5 \mathrm{~s}\}$ via (2.5), as in Sect. 2.3.3. The non-zero stress signals will be denoted for each case based on (2.6), 


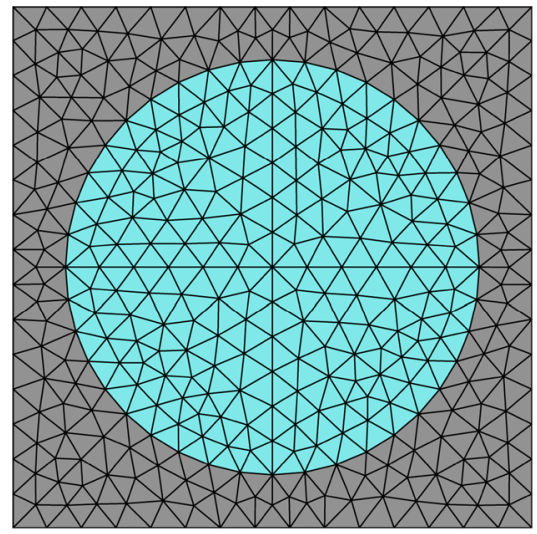

(a) Microstructure

Fig. 13 The microstructure geometry and the loading scenario $\left(\bar{\epsilon}_{12} \neq\right.$ 0 ) are depicted for the M2C1 setup of Sect. 4.2.1. Here, as well as in similar figures which follow, $E^{(2)}$ is associated with the turquoise constituent while the remaining constituent is assigned $E^{(1)}$. Presently,

$T_{\sigma}=T_{\epsilon}$ being the common choice unless otherwise noted. Isotropic linearly elastic constituents are assumed and each is assigned a zero Poisson's ratio for simplicity, following the example of Sect. 3.2, leaving the Young's modulus as the only material parameter that is either fixed or variable. Note that the simple influence of the microstructure in rendering the macroscopic stress nonlinear with respect to the variable moduli was demonstrated in Sects. 2.4.3 and 3.2. For the more complex microstructure geometries to be considered in this section, this relation is again intrinsically nonlinear. Moreover, the Poisson effect is non-zero on the macroscale in the presence of a non-trivial microstructure despite zero microscopic Poisson's ratios, which leads to full coupling among stress and strain components on the macroscale. The developed control framework is able to address this coupling, which will be demonstrated in Sect. 4.2.2.

In all $d$-dimensional examples in a general MIMO setting, the number of input variables $n$ of the control loop is equal to the number of output variables. The mechanical (M) dimension $d$ governs the overall cost of the simulation whereas the control $(\mathrm{C})$ variable number $n$ governs the complexity of the control problem. The notation $\mathrm{M} d \mathrm{Cn}$ will therefore be employed as an indicator for the challenge associated with the particular problem of tunable mechanics. Note that the example in Sect. 3.2 already highlighted the importance of embedding tunable mechanics within a properly constructed microstructure in order to enable control towards target signals in complex loading scenarios. In the examples that follow, representative microstructures will be employed which already provide a suitable adaptation space for the prescribed problem. The FEM mesh resolution for these microstructures will be indicated in corresponding

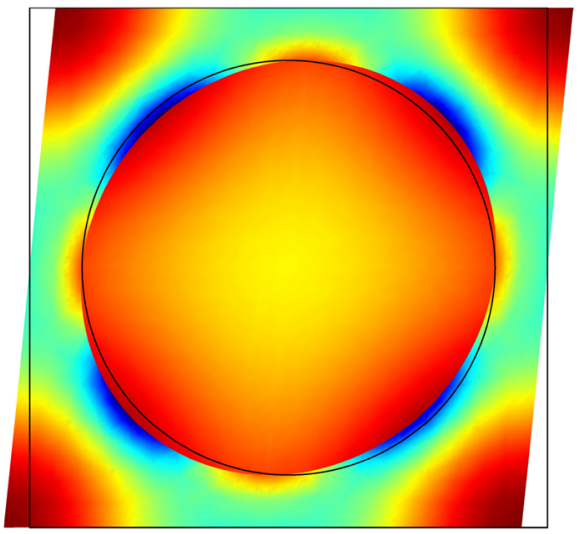

(b) Loading

$f^{(1)}=f^{(2)}=0.5$ and only the particle elastic modulus $E^{(2)}$ is variable. The color distribution on the deformed configuration (scaled, at an instant of loading) is shown as an indicator for the magnitude of the shear stress, with red corresponding to maximum and blue corresponding to minimum value. (Color figure online)

figures which summarize the setup. The time resolution is fixed in all examples such that a period is traversed with $10^{4}$ steps. The underlying controllers follow the presentation of Sect. 3.

\subsection{Two-dimensional mechanics}

\subsubsection{One-variable control (M2C1)}

For M2C1, the particulate microstructure in Fig. 13 is considered (see also Fig. 2). The unit-cell is subjected to shear, where the target stress signal $\bar{\sigma}_{12}^{*}$ is described by the default signal from Fig. 4 and the matrix is assigned the fixed property $E^{(1)}=150 \mathrm{MPa}$. Note that a very large value for $E^{(1)}$ will easily render the target path unrealizable due to the high shear stiffness that is provided by the matrix material alone. With the chosen setup, on the other hand, the microstructure can adapt to the control demands and the tracking error quickly diminishes below one percent (Fig. 14). The fact that the tracking error saturates to a non-zero value follows from the delay and filter components of the control framework. However, this value is sufficiently small so as to deliver virtually overlapping actual and target stress signals beyond the first few cycles.

\subsubsection{Two-variable control (M2C2)}

For M2C2, the microstructure in Fig. 15 is considered. The unit-cell is subjected to biaxial loading, where the target stress signals are borrowed from Fig. 12. Note that the microstructure geometry is chosen so as to ensure both target signals are realizable when both microscopic constituents 


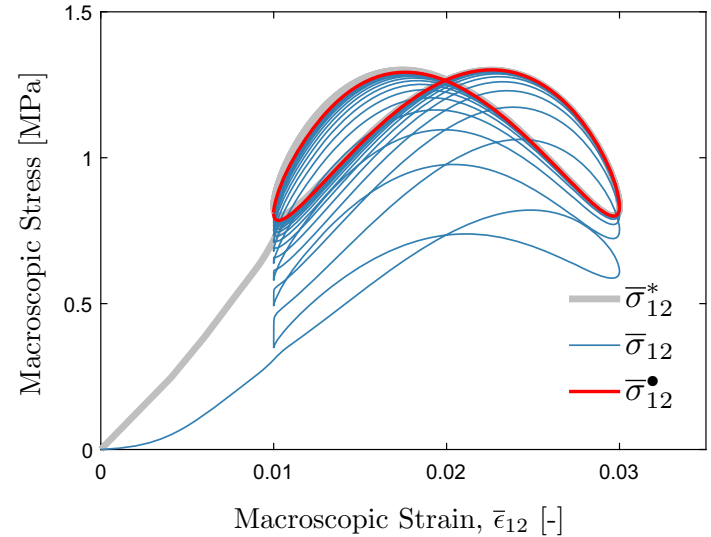

(a) Macroscopic path

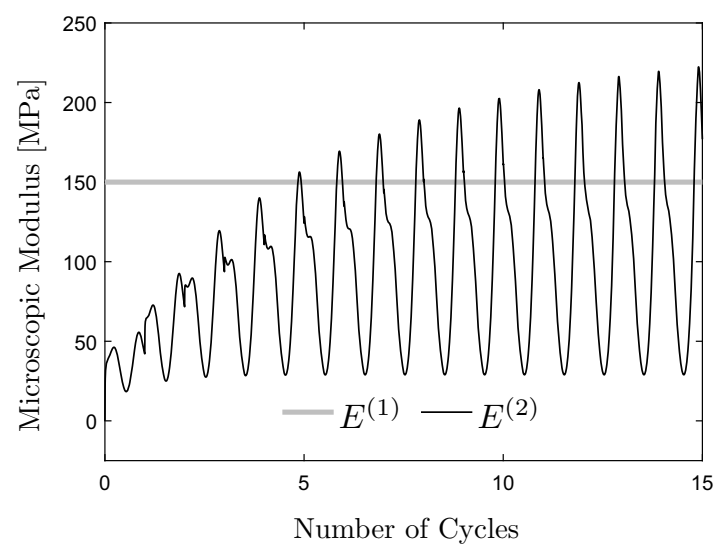

(c) Microscopic modulus

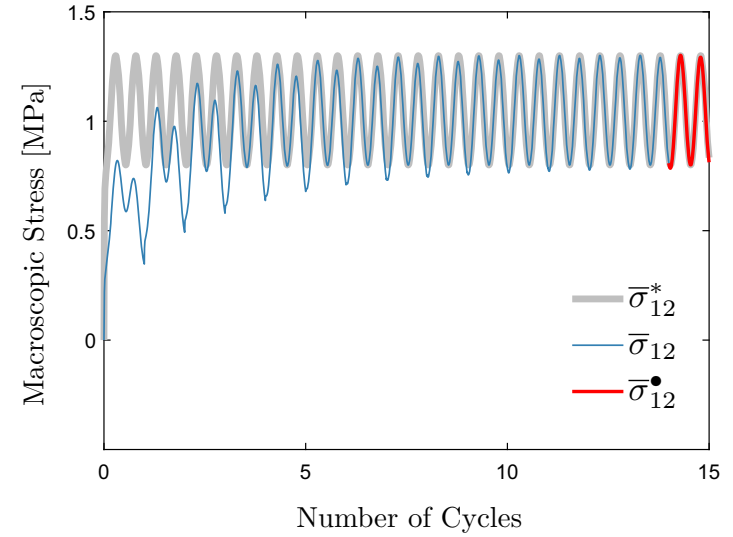

(b) Macroscopic stress

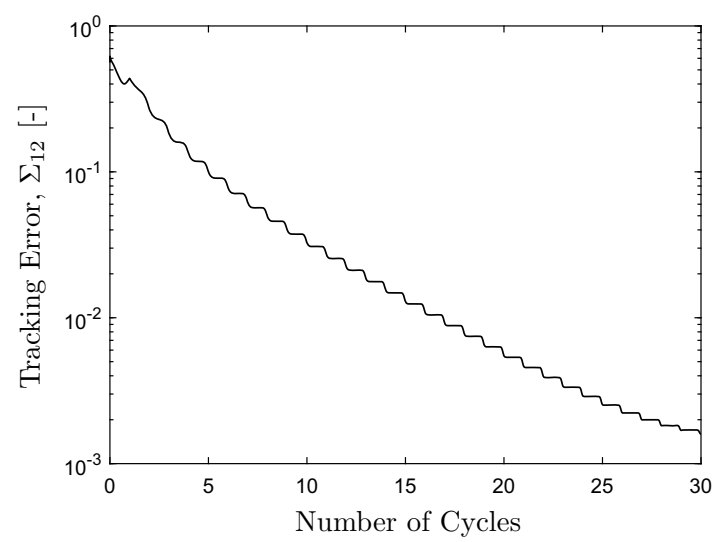

(d) Tracking error

Fig. 14 The controller performance is demonstrated for the M2C1 setup of Sect. 4.2.1. Here, $\bar{\sigma}_{12}^{*}$ is constructed using $c y c=\cos$ with $T_{\sigma}=T_{\epsilon} / 2$ and $\theta=\pi / 3$ (see Fig. 3)

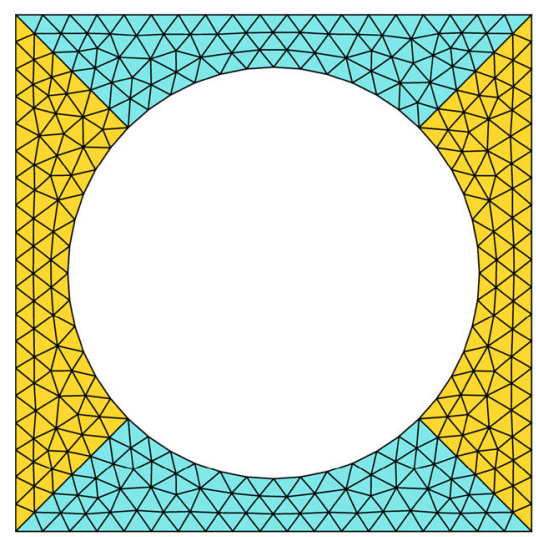

(a) Microstructure

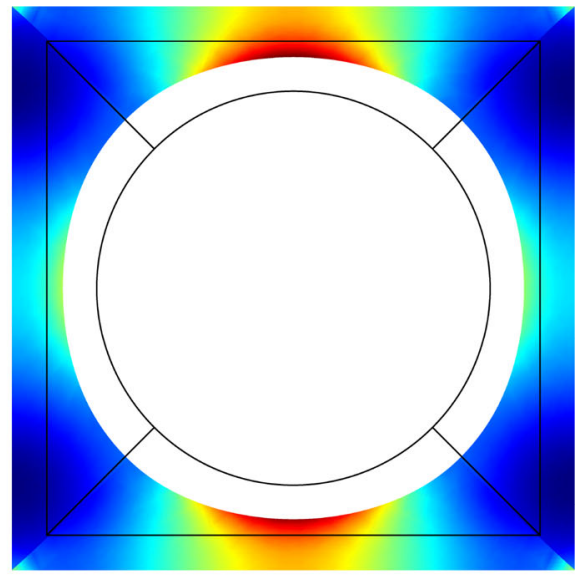

(b) Loading
Fig. 15 The microstructure geometry and the loading scenario $\left(\bar{\epsilon}_{11} \neq 0\right.$ and $\bar{\epsilon}_{22} \neq 0$ ) are depicted for the M2C2 setup of Sect. 4.2.2. Both constituents are tunable with a cell fraction $f^{(1)}=f^{(2)}=0.25$, each contributing predominantly to the stress component along its individual axis of orientation. The color distribution on the deformed configura- tion (scaled, at an instant of loading) is shown as an indicator for the magnitude of the equivalent (von Mises) stress, with red corresponding to maximum and blue corresponding to minimum value. (Color figure online) 


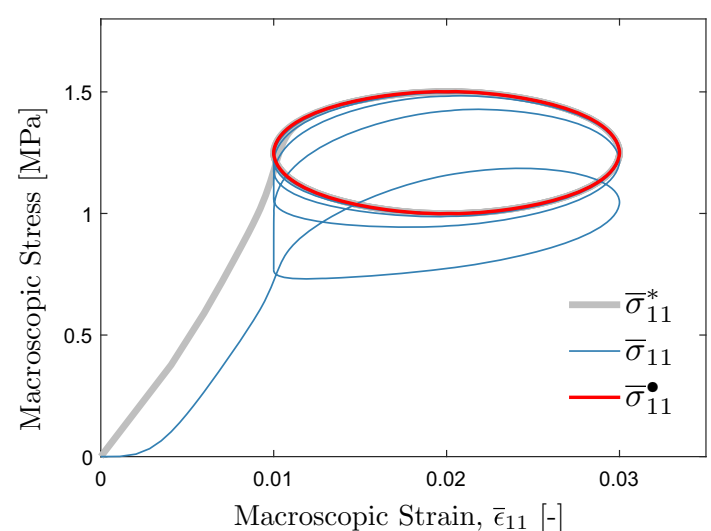

(a) Macroscopic path

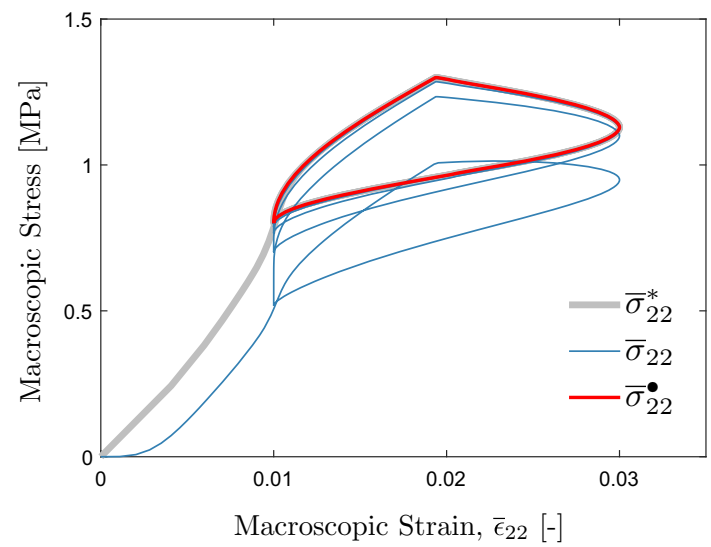

(c) Macroscopic path

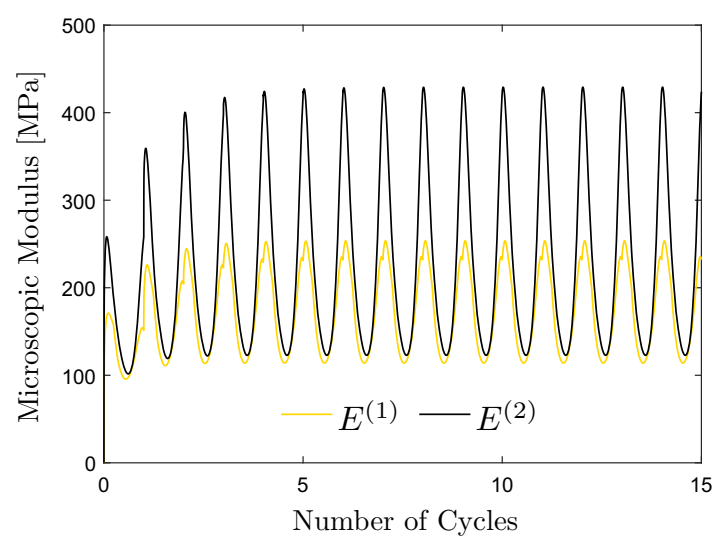

(e) Microscopic modulus

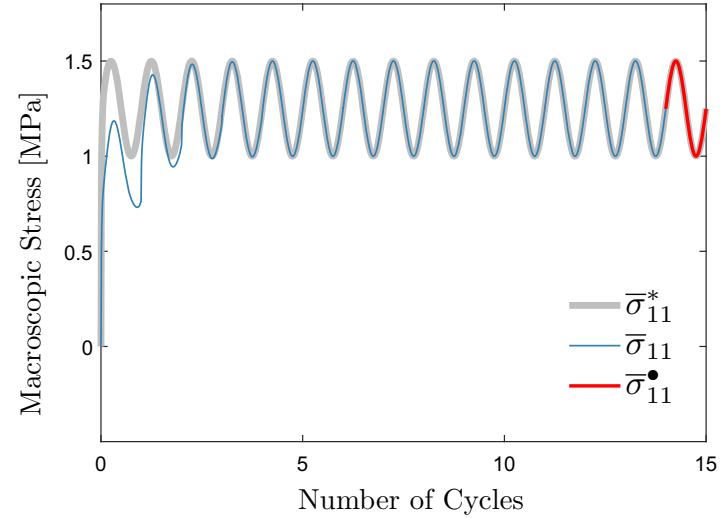

(b) Macroscopic stress

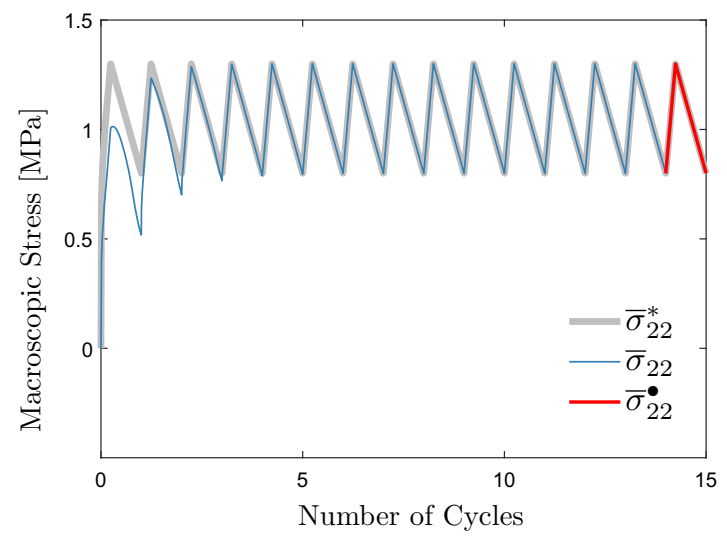

(d) Macroscopic stress

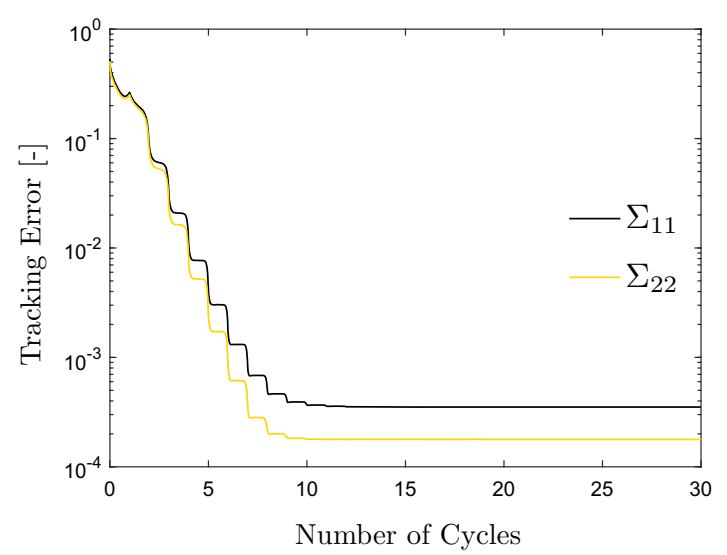

(f) Tracking error

Fig. 16 The controller performance is demonstrated for the M2C2 setup of Sect. 4.2.2

are tunable. Indeed, the results in Fig. 16 demonstrate a performance that is similar to the former M2C2 example of Sect. 3.2. However, unlike this earlier example, the present microstructure necessitates the numerical determination of the microscopic stress distribution and therefore requires a larger computation time to generate the summarized results with the given FEM mesh. Clearly, despite the identical macroscopic strain variation along both directions, the entirely independent macroscopic stress variations are an indication of the non-conventional anisotropic macroscopic response that goes beyond the expected behavior for the geometrically orthogonal symmetry of this microstructure, thereby further highlighting the possibilities enabled by tunable composites. 
Fig. 17 The microstructure geometry and the loading scenario $\left(\bar{\epsilon}_{13} \neq 0\right)$ are depicted for the M3C1 setup of Sect. 4.3. The pore $\left(E^{(1)}=0\right)$ leaves the matrix elastic modulus $E^{(2)}$ as the only variable with $f^{(1)}=f^{(2)}=0.5$. The color distribution on the deformed configuration (scaled, at an instant of loading) is shown as an indicator for the magnitude of the shear stress, with red corresponding to maximum and blue corresponding to minimum value. (Color figure online)

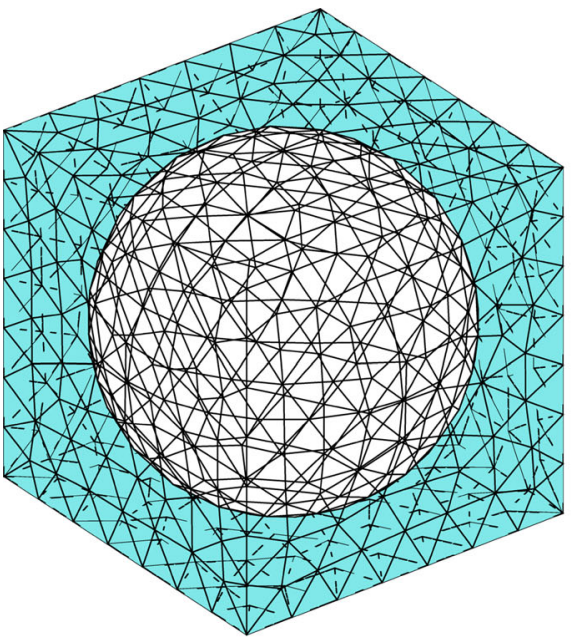

(a) Microstructure

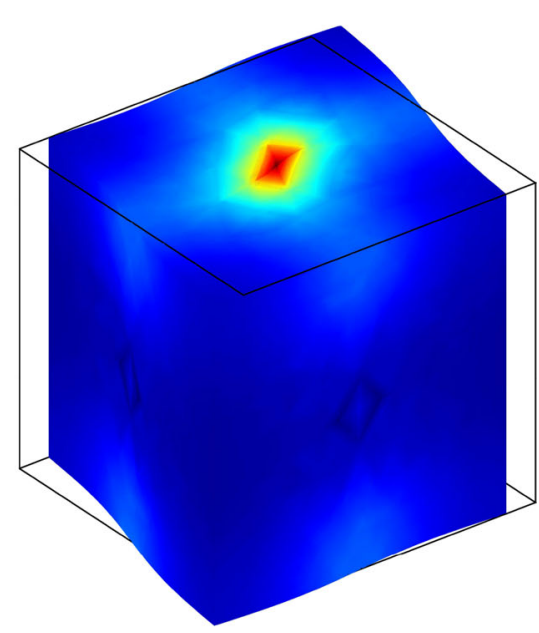

(b) Loading

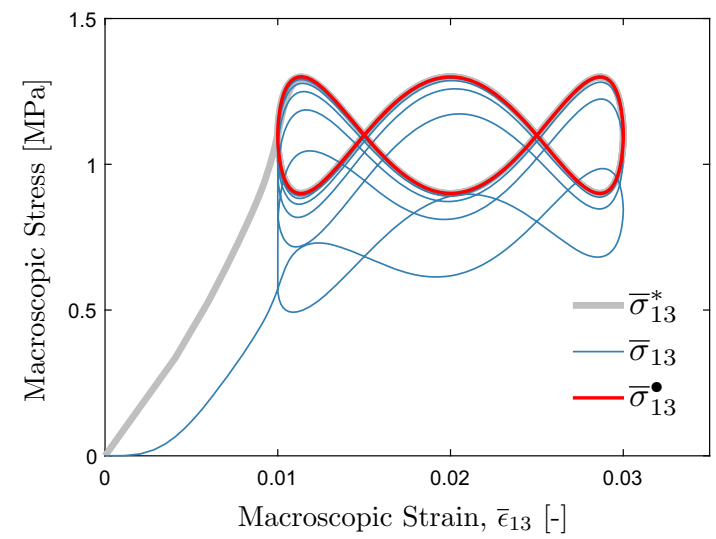

(a) Macroscopic path

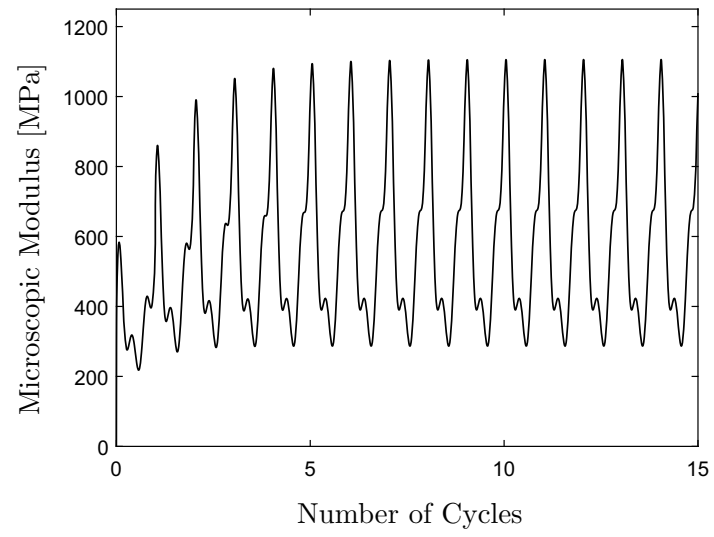

(c) Microscopic modulus $E^{(2)}\left(E^{(1)}=0\right)$

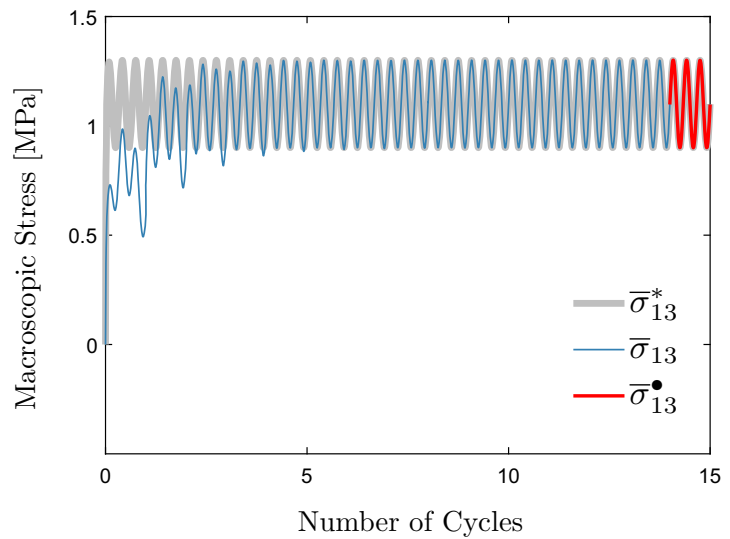

(b) Macroscopic stress

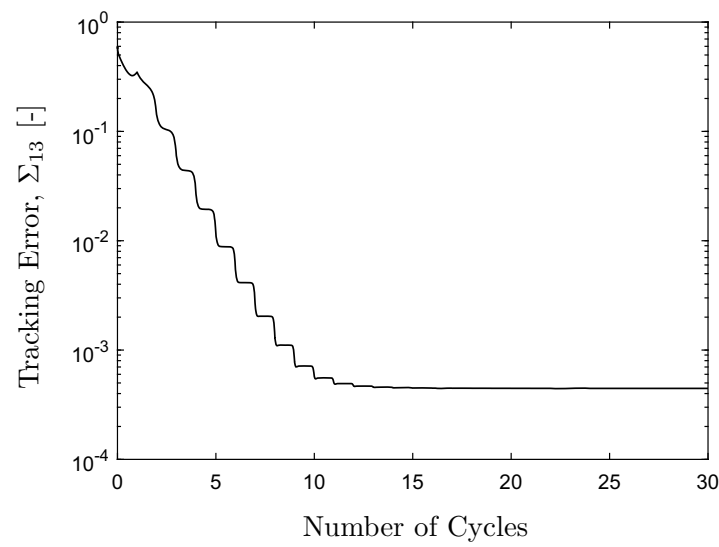

(d) Tracking error

Fig. 18 The controller performance is demonstrated for the M3C1 setup of Sect. 4.3. Here, $\bar{\sigma}_{13}^{*}$ is constructed using $\operatorname{cyc}=\cos$ with $T_{\sigma}=T_{\epsilon} / 3$ and $\theta=\pi / 2$ (see Fig. 3) as well as $\left\{\bar{\sigma}_{o}, \Delta \bar{\sigma}\right\}=\{1.1 \mathrm{MPa}, 0.2 \mathrm{MPa}\}$ in (2.6)

Recalling the challenge that was pointed out in Sect. 3.2, a discussion of the M2C3 setup will presently not be attempted with more complex microstructures. Instead, the feasibility of tunable mechanics in a three-dimensional setting will be demonstrated next. 


\subsection{Three-dimensional mechanics}

As a three-dimensional example, the $\mathrm{M} 3 \mathrm{C} 1$ setup in Fig. 17 is considered. Here, for notational convenience, $E^{(1)}=0$ is assigned to indicate the porous nature of the microstructure. Despite the fact that the problem targets a single shear stress signal by tuning a single microscopic elastic material via $E^{(2)}$, the microstructure still plays a role because its macroscopic response is anisotropic although the microscopic material is isotropic. The transition from a two-dimensional setting to a three-dimensional one can significantly increase the simulation cost, depending on the numerical resolution in time and space. In view of the fact that, with a suitably constructed controller, the number of control variables $n$ does not by itself lead to a significant change in the simulation time, the successful results in Fig. 18 already demonstrate the feasibility of the computational framework in a threedimensional setting. Clearly, for the same problem, different pore morphologies can easily enhance or inhibit the ease with which control is carried out, for instance by altering the range over which $E^{(2)}$ is varied. Consequently, this example also demonstrates the importance of the choice of the microstructure that was pointed out earlier in Sects. 3.2 and 4.2.2.

\section{Conclusion}

The goal of this work was to explore smart composites which display tunable consitutive behavior that enables them to exhibit nearly optimal behavior under time-varying performance criteria. This goal was carried out in a numerical setting via three major steps: (1) the demonstration of the possibilities offered by such composites, which sets the demands on the numerical approach, (2) the development of controllers that are appropriate for mechanics in multiple dimensions, and (3) the integration of the control approach within a general computational method in order to address realistic microstructures. In view of the envisioned practical applications of this work, adaptation through tuning was sought for periodic signals and was realized through repetitive controllers for which performance demonstrations were presented. Various examples indicated the success with which these controllers enable smart response such that complex paths in stress-strain space could be followed with high precision in the availability of mediocre tunability, where complexity primarily refers to the qualitative fact that no combination of traditional materials can display such a behavior. Finally, despite the need for a large number of micromechanical simulations throughout the tuning effort, the feasibility of working in a fully three-dimensional setting was also demonstrated.

A number of challenges form a basis for future work. Among these, the experimental study with such compos- ites is an outstanding one, with respect to material selection, composite manufacturing and controller design. The numerical framework developed presently certainly forms a starting point for the proper design and control of a smart composite, although further effort is needed in order to address practical difficulties such as microstructural defects or feedback delays from the sensors. From a pure computational point of view, another outstanding challenge is addressing the strong interaction between the microstructure topology and the freedom in adaptation, especially with respect to ensuring realizable target signals in a multi-dimensional multi-inputmulti-output setting. Here, one can seek to design the microstructure so as to endow it with maximum tunability, which will translate into a clear identification of how to distribute each microscopic constituent so as to follow all target signals with high precision. These possibilities, among others, highlight a rich spectrum of open issues that lie in this novel field and addressing these will particularly benefit from recent developments in computational mechanics, additive manufacturing and control theory towards the design, manufacturing and operation of smart composite systems.

\section{References}

1. Kaw AK (2005) Mechanics of composite materials, 2nd edn. CRC Press, Boca Raton

2. Zohdi TI (2003) Constrained inverse formulations in random material design. Comput Methods Appl Mech Eng 192:3179-3194

3. Guest JK (2015) Optimizing the layout of discrete objects in structures and materials: a projection-based topology optimization approach. Comput Methods Appl Mech Eng 283:330-351

4. Kiyono CY, Silva ECN, Reddy JN (2012) Design of laminated piezocomposite shell transducers with arbitrary fiber orientation using topology optimization approach. Int J Numer Methods Eng 90(12): 1452-1484

5. Khani A, Abdalla MM, Gürdal Z (2015) Optimum tailoring of fibresteered longitudinally stiffened cylinders. Compos Struct 122:343351

6. Lee J-H, Singer JP, Thomas EL (2012) Micro-/nanostructured mechanical metamaterials. Adv Mater 24:4782-4810

7. Bendsøe MP, Sigmund O (2004) Topology optimization: theory, methods and applications, 2nd edn. Springer, Berlin

8. Christensen PW, Klarbring A (2010) An introduction to structural optimization. Springer, Berlin

9. Sigmund O, Torquato S (1996) Composites with extremal thermal expansion coefficients. Appl Phys Lett 69:3203-3205

10. Sigmund O (2000) A new class of extremal composites. J Mech Phys Solids 48:397-428

11. Chen B-C, Silva ECN, Kikuchi N (2001) Advances in computational design and optimization with application to MEMS. Int $\mathbf{J}$ Numer Methods Eng 52(1-2):23-62

12. Nakshatrala PB, Tortorelli DA, Nakshatrala KB (2013) Nonlinear structural design using multiscale topology optimization. Part I: static formulation. Comput Methods Appl Mech Eng 261262:167-176

13. Kato J, Yachi D, Terada K, Kyoya T (2014) Topology optimization of micro-structure for composites applying a decoupling multiscale analysis. Struct Multidiscip Optim 49:595-608 
14. Rafsanjani A, Akbarzadeh A, Pasini D (2015) Snapping mechanical metamaterials under tension. Adv Mater 27:5931-5935

15. Haghpanah B, Salah-Sharif L, Pourrajab P, Hopkins J, Valdevit L (2016) Multistable shape-reconfigurable architected materials. Adv Mater 28:7915-7920

16. Restrepo D, Mankame ND, Zavattieri PD (2016) Programmable materials based on periodic cellular solids. Part I: experiments. Int J Solids Struct 100-101:485-504

17. Shan W, Lu T, Majidi C (2013) Soft-matter composites with electrically tunable elastic rigidity. Smart Mater Struct 22:085005

18. Shan W, Diller S, Tutcuoglu A, Majidi C (2015) Rigidity-tuning conductive elastomer. Smart Mater Struct 24:065001

19. Kallio M (2005) The elastic and damping properties of magnetorheological elastomers. Ph.D. thesis, Tampere University of Technology, Tampere, Finland

20. Li WHG, Zhou Y, Tian TF (2010) Viscoelastic properties of MR elastomers under harmonic loading. Rheol Acta 49:733-740

21. Kallio M, Lindroos T, Aalto S, Järvinen E, Kärnä T, Meinander T (2007) Dynamic compression testing of a tunable spring element consisting of a magnetorheological elastomer. Smart Mater Struct 16:506-514

22. Li Y, Li J, Li W, Du H (2014) A state-of-the-art review on magnetorheological elastomer devices. Smart Mater Struct 23:123001

23. Lee D, Lee M, Jung N, Yun M, Lee J, Thundat T, Jeon S (2014) Modulus-tunable magnetorheological elastomer microcantilevers. Smart Mater Struct 23:055017

24. Kuder IK, Arrieta AF, Raither WE, Ermanni P (2013) Variable stiffness material and structural concepts for morphing applications. Prog Aerosp Sci 63:33-55

25. Churchill CB, Shahan DW, Smith SP, Keefe AC, McKnight GP (2016) Dynamically variable negative stiffness structures. Sci Adv 2:e1500778

26. Jackson JA, Messner MC, Dudukovic NA, Smith WL, Bekker L, Moran B, Golobic AM, Pascall AJ, Duoss EB, Loh KJ, Spadaccini CM (2018) Field responsive mechanical metamaterials. Sci Adv 4:eaau6419

27. Roh S, Okello LB, Golbasi N, Hankwitz JP, Liu JAC, Tracy JB, Velev OD (2019) 3D-printed silicone soft architectures with programmed magneto-capillary reconfiguration. Adv Mater Technol 4(4):1800528
28. Pavliotis GA, Stuart AM (2008) Multiscale methods: averaging and homogenization. Springer, Berlin

29. Torquato S (2002) Random heterogeneous materials: microstructure and macroscopic properties. Springer, Berlin

30. Zohdi TI, Wriggers P (2005) Introduction to computational micromechanics. Springer, Berlin

31. Ogata K, Yang Y (2002) Modern control engineering, 4th edn. Prentice Hall, London

32. Desoer CA, Vidyasagar M (1975) Feedback systems: input-output properties, vol 55. SIAM, Philadelphia

33. Zhou K, Doyle JC, Glover K (1996) Robust and optimal control. Prentice Hall, Philadelphia

34. Chen CT (2013) Linear system theory and design. Oxford series in electrical and computer engineering. Oxford University Press, Oxford

35. Özcan M (2018) Smart composites with tunable stress-strain curves. Master's thesis, Bilkent University, Bilkent, Turkey

36. Hara S, Omata T, Nakano M (1985) Synthesis of repetitive control systems and its application. In: Decision and control, 1985 24th IEEE conference on, vol 24, pp 1387-1392. IEEE

37. Francis BA, Wonham WM (1975) The internal model principle of linear control theory. IFAC Proc Vol 8(1):331-336

38. Wang Y, Gao F, Doyle FJ (2009) Survey on iterative learning control, repetitive control, and run-to-run control. J Process Control 19(10):1589-1600

39. Hara S, Yamamoto Y, Omata T, Nakano M (1988) Repetitive control system: a new type servo system for periodic exogenous signals. IEEE Trans Autom Control 33(7):659-668

Publisher's Note Springer Nature remains neutral with regard to jurisdictional claims in published maps and institutional affiliations. 